\title{
Analysis of DF Relay Selection in Massive MIMO Systems With Hardware Impairments
}

\author{
Mohammad Kazemi ${ }^{\circledR}$, Member, IEEE, Abbas Mohammadi ${ }^{\circledR}$, Senior Member, IEEE, \\ and Tolga M. Duman ${ }^{\circledR}$, Fellow, IEEE
}

\begin{abstract}
We consider a massive multiple-input multiple-output (m-MIMO) system in which a source communicates with a destination with the help of multiple single-antenna decode-and-forward (DF) relays. Employing optimal relay selection, we analyze the system performance in presence of hardware impairments (HWI) for two m-MIMO scenarios: massive-antenna source and singleantenna destination (m-MIMO I), and massive-antenna source and destination (m-MIMO II). We obtain lower bounds on the average signal-to-noise plus distortion ratio (SNDR) of the system and show that in the m-MIMO II regime, the HWI levels at the relays become the only limiting factors. Employing extreme value theory, we demonstrate that as the number of relays increases the end-to-end SNDR of the system tends to Gumbel and Weibull distributions for the m-MIMO I and m-MIMO II systems, respectively. In addition, for both arbitrary numbers of source and destination antennas and m-MIMO scenarios, we provide closed form expressions for optimal power allocation between the source and the selected relay, and the effects of HWI level distributions between the receiving and the transmitting parts of the relay (which can be exploited for optimal system design under cost constraints).
\end{abstract}

Index Terms-DF relay selection, hardware impairments, massive MIMO, extreme value theory, scalability.

\section{INTRODUCTION}

$\mathbf{N}$ UMEROUS advantages of massive multiple-input multiple-output (m-MIMO) systems have made them one of the key technology enablers for the next generation of wireless communication systems [2]-[4]. In order to reduce the cost in m-MIMO systems, due to the high number of RF elements, it is desirable to use cheap hardware components such as one-bit analog-to-digital converters (e.g., [5]) and one-bit digital phase shifters alongside a common power amplifier [6]. Utilizing cheap hardware components increases the hardware impairments (HWI), which may degrade the overall system performance. A commonly adopted model for $\mathrm{HWI}$ in $5 \mathrm{G}$

Manuscript received July 17, 2019; revised November 17, 2019; accepted December 22, 2019. Date of publication January 23, 2020; date of current version June 18,2020 . The work of M. Kazemi and A. Mohammadi was supported by Iran National Science Foundation (INSF) under Agreement 96007127. This paper was presented in part at the International Symposium on Telecommunication (IST), Tehran, Iran, Dec. 17-19, 2018 [1]. The review of this article was coordinated by Dr. N.-D. Dao. (Corresponding author: Mohammad Kazemi.)

Mohammad Kazemi and Abbas Mohammadi are with the Department of Electrical Engineering, Amirkabir University of Technology (Tehran Polytechnic), Tehran 15875-4413, Iran (e-mail: mohammadkaazemi@aut.ac.ir; abm125@aut.ac.ir).

Tolga M. Duman is with the Department of Electrical and Electronics Engineering, Bilkent University, Ankara 06800, Turkey (e-mail: duman@ee.bilkent.edu.tr).

Digital Object Identifier 10.1109/TVT.2020.2968874 systems is the additive stochastic impairment model in [7], [8], in which the residual impairments after compensation are treated as additive Gaussian noise whose variance is proportional to transmit and receive powers.

On a different front, in future communication systems, cooperative relaying will play an important role in increasing the system throughput and coverage [21]-[23], where the relay nodes can be user nodes (not necessarily a node dedicated to relaying). As the number of available relays increases, relay selection will also become an integral part of an overall wireless communication system. With this motivation, our focus in this paper is on massive MIMO systems with cooperative transmission and hardware impairments.

There are some existing results on the effects of residual HWI on cooperative systems with relay selection [9]-[16]. The performance of two-way amplify-and-forward (AF) relaying with the best relay in the presence of HWI is analyzed in [9]. In [10], the authors derive closed-form and asymptotic expressions for the outage probability of dual-hop AF relaying networks with HWI over Rayleigh fading channels. The authors extend their work to both AF and decode-and-forward (DF) relays over shadowedRician channels in [11], and also to two-way multiple-antenna relays in [12]. In [13], the performance of a DF relaying system with energy harvesting relays is analyzed with two selection schemes: selection of the relay which potentially harvests the highest amount of energy from the source signal and selection of the relay which maximizes the end-to-end signal-to-noise plus distortion ratio (SNDR). In [14], the authors investigate the outage performance of a secondary AF relaying network in a cognitive radio system in the presence of HWI with the assumption of a direct path between the secondary source and destination. In [15], analytical expressions and upper bounds are derived for the outage probability and ergodic capacity of a dual-hop radio frequency/free-space optical (RF/FSO) system with multiple relays employing DF and AF schemes. In [16], the authors analyze the performance of a DF relaying system with single-antenna relays and multiple-antenna source and destination by considering only the single hop channel gains in the relay selection process.

All the works on the effects of residual HWI on cooperative systems with relay selection, except [16], consider singleantenna source and destination, and none tackles the m-MIMO scenario. In this paper, we analyze the end-to-end performance of a m-MIMO system with multiple DF relays in the presence of residual HWI for two m-MIMO scenarios: massive-antenna source and single-antenna destination, and massive-antenna 


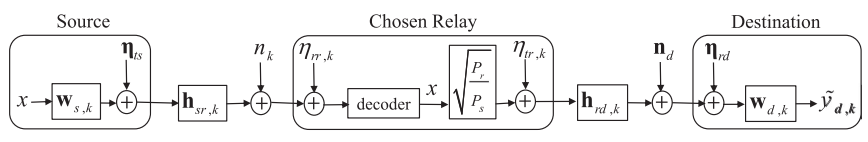

Fig. 1. A DF relay selection system with no direct path.

source and destination. Differently from [16], in our work, we consider the optimal relay selection scheme based on the channel gains of both hops. The contributions of this paper are fourfold:

1) We derive closed-form expressions for the cumulative distribution function (CDF) of the end-to-end SNDR of the system, and obtain lower bounds on the average endto-end SNDR.

2) We derive closed form expressions for the optimal power allocation between the source and the selected relay for both arbitrary numbers of source and destination antennas, and m-MIMO scenarios.

3) We provide closed form expressions for the HWI level distribution between the receiving and the transmitting parts of the relay by formulating a stochastic optimization problem.

4) We show via extreme value theory that, by increasing the number of relays, the normalized end-to-end SNDR tends to Gumbel and Weibull distributions for the m-MIMO I and m-MIMO II systems, respectively. We also obtain closed-form expressions for the normalization coefficients.

The rest of the paper is organized as follows. In Section II, the system model is introduced. A closed-form expression for the CDF of the end-to-end SNDR and a lower bound on its average are derived in Section III. Details on optimal power and HWI level allocations are given in Sections IV and V, respectively. The scalability analysis is presented in Section VI. Numerical examples are provided in Section VII, and finally, the paper is concluded in Section VIII.

\section{SySTEM ModeL}

The general system model consists of a source with $N_{s}$ antennas communicating with a destination with $N_{d}$ antennas with the help of $K$ single-antenna DF relays, which operate in half-duplex mode. Clearly, utilizing single antenna relays is not a bottleneck since we are employing multiple relays and a corresponding relay selection scheme, making sure that MIMO gains are available for the overall system. We focus on two m-MIMO scenarios: 1) when only the source is equipped with massive number of antennas and the destination has a single antenna, which models communications between a base station and an end user, 2) when both the source and the destination are equipped with massive numbers of antennas, which models communications between two base stations. We refer to these scenarios as $m$-MIMO I and $m$-MIMO II, respectively. We also assume that all the channel gains are independent of each other and perfectly available at the source (both hops) and the destination (only second hop) [17], [18], and that there is no direct path between the source and destination. The system model is depicted in Fig. 1, where $R_{b}$ is the selected relay.

The transmission takes place in two phases. In the first phase, employing maximal ratio transmission (MRT) precoding, the source sends data to the relay with the highest end-to-end SNDR. Hence, in the presence of HWI, the received signal at the $k$ th relay (if it is the chosen one), $y_{r, k}$, for the first hop can be written as

$$
y_{r, k}=\left(\mathbf{w}_{s, k} x+\boldsymbol{\eta}_{t s}^{\mathrm{T}}\right) \mathbf{h}_{s r, k}+\eta_{r r, k}+n_{k},
$$

where $x$ is the transmitted symbol, $n_{k}$ is circularly-symmetric complex zero mean additive white Gaussian noise (AWGN) with variance $\sigma_{n}^{2}$ (i.e., $n_{k} \sim \mathcal{C N}\left(0, \sigma_{n}^{2}\right)$ ) at the $k$-th relay, $\mathbf{h}_{s r, k}$ is an $N_{s} \times 1$ vector of channel coefficients between the source and the $k$-th relay, which is modeled as a circularlysymmetric complex Gaussian vector with independent, zero mean and unit variance entries (i.e., $\mathbf{h}_{s r, k} \sim \mathcal{C N}\left(\mathbf{0}, \mathbf{I}_{N_{s}}\right)$ ), and $\mathbf{w}_{s, k} \triangleq \frac{\mathbf{h}_{s r, k}^{\mathrm{H}}}{\left\|\mathbf{h}_{s r, k}\right\|}$ is the $1 \times N_{s}$ MRT precoding vector for the $k$-th relay, where $\|\cdot\|$ denotes the Euclidean norm. In (1), $\boldsymbol{\eta}_{t s} \sim$ $\mathcal{C N}\left(\mathbf{0}, \frac{\kappa_{t s}^{2} P_{s}}{\left\|\mathbf{h}_{s r, k}\right\|^{2}} \operatorname{diag}\left(\left|h_{s r, k}^{(1)}\right|^{2}, \ldots,\left|h_{s r, k}^{\left(N_{s}\right)}\right|^{2}\right)\right)$, where $\operatorname{diag}(\mathbf{x})$ denotes a diagonal matrix with the elements of $\mathbf{x}$ on its diagonal, and $\eta_{r r, k} \sim \mathcal{C N}\left(0, \kappa_{r r, k}^{2} P_{s}\left\|\mathbf{h}_{s r, k}\right\|^{2}\right)$ models the residual HWI in the source's transmitter and those in the $k$-th relay's receiver, respectively, with $P_{s}=\mathrm{E}\left[|x|^{2}\right]$. The coefficients $\kappa_{t s}>0$ and $\kappa_{r r, k}>0$ characterize the HWI levels at the source's transmitter and the $k$-th relay's receiver, respectively [28], [29].

In the second phase, the chosen relay decodes and re-encodes the received data and sends it to the destination. The received signal at the destination, $\mathbf{y}_{d, k}$, can be written as

$$
\mathbf{y}_{d, k}=\left(\sqrt{\frac{P_{r}}{P_{s}}} x+\eta_{t r, k}\right) \mathbf{h}_{r d, k}+\boldsymbol{\eta}_{r d}+\mathbf{n}_{d},
$$

where $P_{r}$ is the average transmit power of the selected relay, $\mathbf{n}_{d}$ is an $N_{d} \times 1$ circularly-symmetric complex Gaussian vector of input noise at the receiver with zero mean and variance $\sigma_{n}^{2}$ entries (i.e., $\mathbf{n}_{d} \sim \mathcal{C N}\left(\mathbf{0}, \sigma_{n}^{2} \mathbf{I}_{N_{d}}\right)$ ), and $\mathbf{h}_{r d, k}$ is the $N_{d} \times 1$ vector of channel coefficients between the $k$-th relay and the destination, which is also modeled as $\mathbf{h}_{r d, k} \sim \mathcal{C N}\left(\mathbf{0}, \mathbf{I}_{N_{d}}\right)$. In (2), $\eta_{t r, k} \sim \mathcal{C N}\left(0, \kappa_{t r, k}^{2} P_{r}\right)$. The $N_{d} \times 1$ vector $\boldsymbol{\eta}_{r d} \sim$ $\mathcal{C N}\left(\mathbf{0}, \kappa_{r d}^{2} P_{r} \operatorname{diag}\left(\left|h_{r d, k}^{(1)}\right|^{2}, \ldots,\left|h_{r d, k}^{\left(N_{d}\right)}\right|^{2}\right)\right)$ models the residual HWI in the $k$-th relay 's transmitter and the destination's receiver, respectively, where $\kappa_{t r, k}>0$ and $\kappa_{r d}>0$ characterize the HWI levels at the $k$-th relay's transmitter and the destination's receiver, respectively [28], [29]. Finally, the destination utilizes maximal ratio combining (MRC) to make decisions on the transmitted symbols.

\section{SNDR ANALYSIS}

In this section, we first obtain closed-form expressions for the SNDRs of the two hops separately, and the CDF of the end-toend SNDR. Then, using these results, we present a lower bound on the average end-to-end SNDR.

Eq. (1) can be divided into the desired signal, distortion and noise parts as follows

$$
y_{r, k}=\underbrace{\mathbf{w}_{s, k} x \mathbf{h}_{s r, k}}_{\text {desired signal }}+\underbrace{\boldsymbol{\eta}_{t s}^{\mathrm{T}} \mathbf{h}_{s r, k}+\eta_{r r, k}}_{\text {distortion }}+\underbrace{n_{k}}_{\text {noise }} .
$$


Since the source utilizes MRT precoding to send data to the selected relay, the SNDR at the $k$-th relay, $\gamma_{s r, k}$, is obtained after some calculations as

$$
\begin{aligned}
\gamma_{s r, k} & =\frac{\left|\mathbf{w}_{s, k} \mathbf{h}_{s r, k}\right|^{2} \mathrm{E}\left[|x|^{2}\right]}{\mathrm{E}\left[\left|\boldsymbol{\eta}_{t s}^{\mathrm{T}} \mathbf{h}_{s r, k}+\eta_{r r, k}\right|^{2}\right]+\mathrm{E}\left[\left|n_{k}\right|^{2}\right]} \\
& =\frac{P_{s}\left\|\mathbf{h}_{s r, k}\right\|^{2}}{P_{s} \alpha_{s r, k}\left\|\mathbf{h}_{s r, k}\right\|^{2}+\sigma_{n}^{2}},
\end{aligned}
$$

where $\alpha_{s r, k}=\kappa_{r r, k}^{2}+\kappa_{t s}^{2} \frac{\left\|\mathbf{h}_{s r, k}\right\|_{4}^{4}}{\left\|\mathbf{h}_{s r, k}\right\|^{4}}$, with $\|\cdot\|_{4}$ denoting the $\ell_{4}$ norm.

For an asymptotic analysis of the SNDR, we need the following two lemmas.

Lemma $1([24, p .148])$ : If $h$ is a zero-mean real-valued Gaussian random variable with variance $\sigma^{2}$, then for any even positive integer $p$, we have $\mathrm{E}\left[h^{p}\right]=\left(\sigma^{2}\right)^{\frac{p}{2}}(p-1) !$ !, where $(\cdot) ! !$ denotes the double factorial, i.e., $(p-1) ! !=1 \times 3 \times \cdots \times$ $(p-1)$.

Lemma 2: If $\mathbf{h}$ is an $N \times 1$ circularly-symmetric complex Gaussian vector with zero mean and unit variance entries, then

$$
\lim _{N \rightarrow \infty} \frac{1}{N}\|\mathbf{h}\|^{2} \stackrel{\text { a.s. }}{\rightarrow} 1 \text { and } \lim _{N \rightarrow \infty} \frac{1}{N}\|\mathbf{h}\|_{4}^{4} \stackrel{\text { a.s. }}{\rightarrow} 2,
$$

where $\stackrel{a . s .}{\rightarrow}$ denotes the almost sure convergence.

Proof: It is readily proved using Lemma 1 and theory of large random vectors [25].

Using Lemma 2 and (4), the maximum possible SNDR of the first hop equals to $\gamma_{s r, k}^{\max }=\lim _{N_{s} \rightarrow \infty} \gamma_{s r, k} \stackrel{a . s .}{\rightarrow} \frac{1}{\kappa_{r r, k}^{2}}$, which only depends on the level of HWI at the relay's receiver.

In the second phase, the selected relay decodes the received signal, re-encodes and transmits it to the destination. At the destination, after applying MRC, we have

$$
\begin{aligned}
\tilde{y}_{d, k}= & \mathbf{w}_{d, k} \mathbf{y}_{d, k} \\
= & \underbrace{\mathbf{w}_{d, k} \sqrt{\frac{P_{r}}{P_{s}}} x \mathbf{h}_{r d, k}}_{\text {desired }}+\underbrace{\mathbf{w}_{d, k} \eta_{t r, k} \mathbf{h}_{r d, k}+\mathbf{w}_{d, k} \boldsymbol{\eta}_{r d}}_{\text {distortion }} \\
& +\underbrace{\mathbf{w}_{d, k} \mathbf{n}_{d}}_{\text {noise }},
\end{aligned}
$$

where $\mathbf{w}_{d, k} \triangleq \frac{\mathbf{h}_{r d, k}^{\mathrm{H}}}{\left\|\mathbf{h}_{r d, k}\right\|}$ is the $1 \times N_{d}$ MRC vector for the $k$-th relay. Using (6), after some calculations, the SNDR at the destination, $\gamma_{r d, k}$, is obtained as

$$
\begin{aligned}
\gamma_{r d, k} & =\frac{\frac{P_{r}}{P_{s}}\left|\mathbf{w}_{d, k} \mathbf{h}_{r d, k}\right|^{2} \mathrm{E}\left[|x|^{2}\right]}{\mathrm{E}\left[\left|\mathbf{w}_{d, k} \eta_{t r, k} \mathbf{h}_{r d, k}+\mathbf{w}_{d, k} \boldsymbol{\eta}_{r d}\right|^{2}\right]+\mathrm{E}\left[\left|\mathbf{w}_{d, k} \mathbf{n}_{d}\right|^{2}\right]} \\
& =\frac{P_{r}\left\|\mathbf{h}_{r d, k}\right\|^{2}}{P_{r} \alpha_{r d, k}\left\|\mathbf{h}_{r d, k}\right\|^{2}+\sigma_{n}^{2}},
\end{aligned}
$$

where $\alpha_{r d, k}=\kappa_{t r, k}^{2}+\kappa_{r d}^{2} \frac{\left\|\mathbf{h}_{r d, k}\right\|_{4}^{4}}{\left\|\mathbf{h}_{r d, k}\right\|^{4}}$.

Using Lemma 2 and (7), the maximum possible SNDR at the second hop equals to $\gamma_{r d, k}^{\max }=\lim _{N_{d} \rightarrow \infty} \gamma_{r d, k} \stackrel{a . s .}{\rightarrow} \frac{1}{\kappa_{r r, k}^{2}}$. Clearly, this value depends only on the level of HWI at the relay's transmitter.
Since the overall performance of the system is limited by the performance of the individual hops, the optimal relay selection criterion and the corresponding end-to-end SNDR can be stated as follows

$$
\begin{aligned}
& R_{b}=\arg \max _{k \in[K]} \min \left\{\gamma_{s r, k}, \gamma_{r d, k}\right\}, \\
& \gamma_{\text {tot }}=\max _{k \in[K]} \gamma_{\text {tot }, \mathrm{k}}=\max _{k \in[K]} \min \left\{\gamma_{s r, k}, \gamma_{r d, k}\right\},
\end{aligned}
$$

where $[K]$ denotes the set of all relay indices. In other words, the source chooses the relay with the highest end-to-end-SNDR, or equivalently, the relay with the highest minimum of the SNDRs in the two hops.

Proposition 1: In the m-MIMO I system $\left(N_{s} \rightarrow \infty\right.$, $\left.N_{d}=1\right)$ with DF relay selection, for $0 \leq \gamma$, the CDF of the end-to-end SNDR with the use of the $k$-th relay, $F_{\Gamma_{\min , k}}(\gamma)$, and the CDF of the end-to-end SNDR of the system, $F_{\Gamma_{\max }}(\gamma)$, are given as

$$
F_{\Gamma_{\min , k}}(\gamma)=1-\exp \left(\frac{-\sigma_{n}^{2} \gamma}{P_{r}\left(1-\kappa_{2, k}^{2} \gamma\right)}\right) u\left(\gamma_{k}^{\max }-\gamma\right)
$$

$$
F_{\Gamma_{\max }}(\gamma)=\prod_{k=1}^{K} F_{\Gamma_{\min , k}}(\gamma)
$$

respectively, where $\gamma_{k}^{\max \triangleq} \min \left\{\left(\kappa_{r r, k}^{2}\right)^{-1},\left(\kappa_{t r, k}^{2}+\kappa_{r d}^{2}\right)^{-1}\right\}$, $\kappa_{2, k} \triangleq \sqrt{\kappa_{t r, k}^{2}+\kappa_{r d}^{2}}$, and $\exp (\cdot)$ and $u(\cdot)$ denote the exponential and Heaviside step functions, respectively.

Proof: The proof is given in Appendix A.

Using this proposition, the following theorem provides a lower bound on the average end-to-end SNDR of the m-MIMO I system (which employs relay selection).

Theorem 1: In the m-MIMO I system $\left(N_{s} \rightarrow \infty, N_{d}=1\right)$ with DF relay selection, the average end-to-end SNDR, $\bar{\gamma}_{\text {tot }}^{I}$, is lower bounded by

$$
\begin{aligned}
\bar{\gamma}_{t o t}^{I} \geq \max _{k \in[K]}\{ & \frac{1}{\kappa_{2, k}^{2}}\left(1-\left(1-\kappa_{2, k}^{2} \gamma_{k}^{\max }\right) e^{-x_{k}^{\max }}\right) \\
& \left.-\frac{1}{\kappa_{2, k}^{2}}\left(\varsigma_{k} e^{\varsigma_{k}}\left(E_{1}\left(\varsigma_{k}\right)-E_{1}\left(x_{k}^{\max }+\varsigma_{k}\right)\right)\right)\right\},
\end{aligned}
$$

where $\quad \varsigma_{k} \triangleq \frac{\sigma_{n}^{2}}{\kappa_{2, k}^{2} P_{r}}, \quad x_{k}^{\max } \triangleq \frac{\sigma_{n}^{2} \gamma_{k}^{\max }}{P_{r}\left(1-\kappa_{2, k}^{2} \gamma_{k}^{\max }\right)} \quad$ and $\quad E_{1}(z)=$ $\int_{z}^{\infty} \frac{1}{t} e^{-t} d t$ is the exponential integral function.

Proof: The proof is given in Appendix B.

Theorem 1 provides an insight on the end-to-end SNDR of the m-MIMO I system. For the m-MIMO II system $\left(N_{s} \rightarrow \infty, N_{d} \rightarrow \infty\right)$, we can obtain simpler SNDR expressions, since both the source and the destination are massive. Applying Lemma 2 to (4) and (7), the SNDRs at the first and second hops of the m-MIMO II system are obtained as

$$
\gamma_{\mathrm{sr}, k}^{\mathrm{II}} \stackrel{a . s .}{\rightarrow} \frac{1}{\kappa_{r r, k}^{2}} \text { and } \gamma_{\mathrm{rd}, k}^{\mathrm{II}} \stackrel{a . s .}{\rightarrow} \frac{1}{\kappa_{t r, k}^{2}},
$$


respectively. Substituting (13) into (9), the end-to-end SNDR of the m-MIMO II system becomes

$$
\gamma_{\text {tot }}^{\text {II }} \stackrel{a . s .}{\rightarrow} \max _{k \in[K]} \min \left\{\frac{1}{\kappa_{r r, k}^{2}}, \frac{1}{\kappa_{t r, k}^{2}}\right\} .
$$

According to (13) and (14), in the m-MIMO II regime, the effects of channel coefficients, input noise terms and HWI at the source and destination fade away, making the HWI at the relays the only remaining limiting factor on the system performance.

\section{A. End-to-End SNDR With HWI Level Variations}

According to (14), in the m-MIMO II system, due to massive numbers of antennas at both ends, the instantaneous SNDR is only a function of HWI levels at the relays. However, even assuming that the relays are identical, they will have slightly different specifications in practice. In this subsection, we assume that there are HWI level variations among the relays, which are modeled statistically. Since there is no characterization and modeling of these variations in the literature, as a starting point, we assume a basic distribution for the variations in HWI levels. More precisely, we assume that the HWI levels at the receiving and transmitting parts of the relays are realizations from i.i.d. uniformly distributed random variables; i.e., $\kappa_{r r}, \kappa_{t r} \sim \mathcal{U}\left(\bar{\kappa}-\frac{\delta_{\kappa}}{2}, \bar{\kappa}+\frac{\delta_{\kappa}}{2}\right)$, where $\bar{\kappa}$ and $\delta_{\kappa}$ are the average HWI level and the range of HWI level variations at the receiving and transmitting parts of the relays. Note that HWI levels of the relays do not change over time.

Theorem 2: In the m-MIMO II system $\left(N_{s} \rightarrow \infty, N_{d} \rightarrow \infty\right)$ with DF relay selection and random, i.i.d., uniformly distributed HWI levels at the relays; i.e., $\kappa_{r r}, \kappa_{t r} \sim \mathcal{U}\left(\bar{\kappa}-\frac{\delta_{\kappa}}{2}, \bar{\kappa}+\frac{\delta_{\kappa}}{2}\right)$, the CDF of the end-to-end SNDR of each relay, $F_{\Gamma_{\min }}^{I I}(\gamma)$ for $\left(\bar{\kappa}+\frac{\delta_{\kappa}}{2}\right)^{-2} \leq \gamma \leq$ $\left(\bar{\kappa}-\frac{\delta_{\kappa}}{2}\right)^{-2}$, is given as follows

$$
F_{\Gamma_{\text {min }}}^{I I}(\gamma)=1-\frac{1}{\delta_{\kappa}^{2}}\left(\frac{1}{\sqrt{\gamma}}-\bar{\kappa}+\frac{\delta_{\kappa}}{2}\right)^{2} .
$$

Also, the average end-to-end SNDR of the system, $\bar{\gamma}_{t o t}^{I I}$, is lower bounded by

$$
\begin{aligned}
\bar{\gamma}_{\text {tot }}^{I I} \geq & \frac{1}{\bar{\kappa}^{2}}+\frac{2 \delta_{\kappa}}{\bar{\kappa}^{3}}\left(\frac{1}{2}-K I_{K}\left(\frac{1}{2}\right)\right) \\
& +\frac{\delta_{\kappa}^{2}}{\bar{\kappa}^{4}}\left(\frac{1}{4}+K\left(I_{K}(1)-I_{K}\left(\frac{1}{2}\right)\right)\right),
\end{aligned}
$$

where $I_{K}(\alpha) \triangleq \sum_{k=0}^{K-1}\left(\begin{array}{c}K-1 \\ k\end{array}\right)(-1)^{k} \frac{1}{k+\alpha+1}$.

Proof: The proof is given in Appendix C.

In the next two sections, we investigate optimization of the system parameters including the transmission powers and the HWI levels at the relays.

\section{OPTIMAL POWER AllocAtion}

In the previous section, we assumed that the transmit powers of the source and the relays are fixed. However, by optimal power allocation (OPA), a better performance can be achieved under a total power constraint. A common practical scenario is sharing a fixed amount of power $P$ between the source and the $k$-th relay [26], namely using a source power of $\lambda_{k} P$ and the $k$ th relay power of $\left(1-\lambda_{k}\right) P$, where $\lambda_{k} \in(0,1)$ is the power allocation factor. Following this approach, we first solve the power allocation problem for arbitrary numbers of the source and the destination antennas. We then analyze it further in the two m-MIMO regimes.

By substituting $P_{s}$ and $P_{r}$ with $\lambda_{k} P$ and $\left(1-\lambda_{k}\right) P$, respectively, in (4) and (7), and utilizing (9), we observe that optimal power allocation and relay selection are accomplished through the following optimization problems

$$
\begin{aligned}
\gamma_{\text {tot }, k}= & \max _{0<\lambda_{k}<1} \min \left\{\frac{\lambda_{k} P\left\|\mathbf{h}_{s r, k}\right\|^{2}}{\lambda_{k} P \alpha_{s r, k}^{2}\left\|\mathbf{h}_{s r, k}\right\|^{2}+\sigma_{n}^{2}},\right. \\
& \left.\frac{\left(1-\lambda_{k}\right) P\left\|\mathbf{h}_{r d, k}\right\|^{2}}{\left(1-\lambda_{k}\right) P \alpha_{r d, k}^{2}\left\|\mathbf{h}_{r d, k}\right\|^{2}+\sigma_{n}^{2}}\right\}, \\
\gamma_{\text {tot }}=\max _{k \in[K]} \gamma_{\text {tot }, k} . &
\end{aligned}
$$

The optimal value of the power allocation factor in (17) is given by the following theorem.

Theorem 3: In a DF relay selection system in which a constant power $P$ is shared with coefficients $\lambda_{k} \in(0,1)$ and $1-\lambda_{k}$ between the source and the $k$-th relay, respectively, conditioned on the instantaneous channel realizations, the optimal value of the power allocation factor $\lambda_{k}$ is obtained as

$$
\lambda_{k}^{\mathrm{opt}}=\frac{-b_{k}^{\prime}+\sqrt{b_{k}^{\prime 2}-4 a_{k}^{\prime} c_{k}^{\prime}}}{2 a_{k}^{\prime}},
$$

where

$$
\begin{aligned}
& a_{k}^{\prime}=P^{2}\left\|\mathbf{h}_{s r, k}\right\|^{2}\left\|\mathbf{h}_{r d, k}\right\|^{2}\left(\alpha_{s r, k}-\alpha_{r d, k}\right), \\
& b_{k}^{\prime}=P \sigma_{n}^{2}\left(\left\|\mathbf{h}_{s r, k}\right\|^{2}+\left\|\mathbf{h}_{r d, k}\right\|^{2}\right)-a_{k}^{\prime}, \\
& c_{k}^{\prime}=-P\left\|\mathbf{h}_{r d, k}\right\|^{2} \sigma_{n}^{2} .
\end{aligned}
$$

Proof: The proof is given in Appendix D.

Finally, the best relay is selected by solving the optimization problem in (18), which is nothing but the simple task of finding the maximum among the corresponding SNDR values $\gamma_{\text {tot, }}$ 's.

In the m-MIMO I system, the SNDR of the first hop becomes independent of the source's transmit power, so a range of power allocation factors become optimal, among which we choose the one which minimizes the total transmit power of the system, as stated in the following theorem.

Proposition 2: In the m-MIMO I system $\left(N_{s} \rightarrow \infty, N_{d}=1\right)$, the optimal total power minimizing strategy is for the source to send with the lowest possible power, i.e. $P_{s}^{\text {opt }} \rightarrow 0$, and for the relay with the following

$$
P_{r}^{\text {opt }}= \begin{cases}\frac{\sigma_{n}^{2}}{\left(\kappa_{r r, k}^{2}-\alpha_{r d, k}^{2}\right)\left|h_{r d, k}\right|^{2}}, & \text { if } \frac{P\left|h_{r d, k}\right|^{2}}{P \alpha_{r d, k}^{2}\left|h_{r d, k}\right|^{2}+\sigma_{n}^{2}} \geq \frac{1}{\kappa_{r r, k}^{2}}, \\ P, & \text { if } \frac{P\left|h_{r d, k}\right|^{2}}{P \alpha_{r d, k}^{2}\left|h_{r d, k}\right|^{2}+\sigma_{n}^{2}}<\frac{1}{\kappa_{r r, k}^{2}} .\end{cases}
$$

Proof: The proof is given in Appendix D.

According to (14), the end-to-end SNDR of the m-MIMO II system becomes independent of transmit powers of both the source and the relays, hence rendering power allocation unnecessary. 


\section{EFFECTS OF TRANSMITTER AND RECEIVER SIDE HWI LEVELS}

In this section, we are interested in studying the effects of HWIs at the transmitter and receiver sides. The best-case HWI level distribution at each relay is a function of the channel coefficient of the two hops. However, in many practical systems, HWI levels are not tunable and, depending on the budget, are fixed at the system design stage. Therefore, in practice, HWI level allocation should not depend on the specific channel realizations. Nevertheless, as an intermediate step toward a stochastic analysis, we first assume that the channel coefficients are fixed, and determine the best-case scenario for the HWI level allocation (HLA). Then, we evaluate the HWI level distribution over the channel statistics, which provides guidelines for an overall system design under a total cost constraint.

\section{A. Evaluation of HWI Levels With Fixed Channel Coefficients}

As in [27], we take the sum of HWI levels at the receiving and the transmitting parts of each relay as a constant. Letting $\kappa_{r, k} \triangleq \kappa_{r r, k}+\kappa_{t r, k}$ be the total HWI level at the $k$-th relay, we write the HWI levels at the receiving and the transmitting parts of the relay as $\kappa_{r r, k}=\rho_{k} \kappa_{r, k}$ and $\kappa_{t r, k}=\left(1-\rho_{k}\right) \kappa_{r, k}$, respectively. Here, $\rho_{k} \in[0,1]$ is the HLA factor for the $k$-th relay. Substituting these values in (4) and (7), and utilizing (9), the relay selection and HWI level allocation can be jointly addressed through an optimization problem which, similar to the power allocation problem, can be divided into two subsequent problems: a HWI level allocation problem as follows and a simple relay selection one as in (18). Namely,

$$
\begin{aligned}
\gamma_{\text {tot }, k}=\max _{0<\rho_{k}<1} & \min \left\{\frac{P_{s}\left\|\mathbf{h}_{s r, k}\right\|^{2}}{P_{s}\left(\beta_{s r, k}+\rho_{k}^{2} \kappa_{r, k}^{2}\right)\left\|\mathbf{h}_{s r, k}\right\|^{2}+\sigma_{n}^{2}},\right. \\
& \left.\frac{P_{r}\left\|\mathbf{h}_{r d, k}\right\|^{2}}{P_{r}\left(\beta_{r d, k}+\left(1-\rho_{k}\right)^{2} \kappa_{r, k}^{2}\right)\left\|\mathbf{h}_{r d, k}\right\|^{2}+\sigma_{n}^{2}}\right\},
\end{aligned}
$$

where $\beta_{s r, k} \triangleq \kappa_{t s}^{2} \frac{\left\|\mathbf{h}_{s r, k}\right\|_{4}^{4}}{\left\|\mathbf{h}_{s r, k}\right\|^{4}}$ and $\beta_{r d, k} \triangleq \kappa_{r d}^{2} \frac{\left\|\mathbf{h}_{r d, k}\right\|_{4}^{4}}{\left\|\mathbf{h}_{r d, k}\right\|^{4}}$.

We first determine the HWI level allocation for arbitrary numbers of source and destination antennas, and then consider the two m-MIMO regimes.

Theorem 4: In a DF relay selection system in which the $k$-th relay has a constant total HWI level $\kappa_{r, k}$ which can be distributed with coefficients $\rho_{k} \in[0,1]$ and $1-\rho_{k}$ between the receiving and the transmitting parts, respectively, the best-case scenario for the $k$-th relay is achieved with an HLA factor equal to

$$
\rho_{\mathrm{k}}^{\mathrm{bc}}= \begin{cases}0, & \text { if } \varpi_{k} \leq 0, \\ \frac{\varpi_{k}}{2 \kappa_{r, k}^{2}}, & \text { if } 0<\varpi_{k}<2 \kappa_{r, k}^{2}, \\ 1, & \text { if } \varpi_{k} \geq 2 \kappa_{r, k}^{2},\end{cases}
$$

where

$$
\varpi_{k} \triangleq \kappa_{r, k}^{2}+\kappa_{r d}^{2} \frac{\left\|\mathbf{h}_{r d, k}\right\|_{4}^{4}}{\left\|\mathbf{h}_{r d, k}\right\|^{4}}-\kappa_{t s}^{2} \frac{\left\|\mathbf{h}_{s r, k}\right\|_{4}^{4}}{\left\|\mathbf{h}_{s r, k}\right\|^{4}}
$$

$$
+\frac{\frac{\sigma_{n}^{2}}{P_{r}}}{\left\|\mathbf{h}_{r d, k}\right\|^{2}}-\frac{\frac{\sigma_{n}^{2}}{P_{s}}}{\left\|\mathbf{h}_{s r, k}\right\|^{2}} .
$$

Proof: The proof is given in Appendix E.

According to Theorem 4, as the HWI level at the source's transmitter $\left(\kappa_{t s}\right)$ increases, the best-case HWI level at the relay's transmitter $\left(\rho_{k}^{\mathrm{bc}} \kappa_{r, k}\right)$ increases as well, while the best-case HWI level at the relay's receiver $\left(\left(1-\rho_{k}^{\mathrm{bc}}\right) \kappa_{r, k}\right)$ decreases. On the other hand, by increasing the HWI level at the destination's receiver $\left(\kappa_{r d}\right)$, the best-case HWI level at the relay's receiver increases as well, while the best-case HWI level at the relay's transmitter decreases. These results are expected, since due to the bottleneck effect in multihop scenarios, the best performance is obtained when the SNDRs of the two hops are as close to each other as possible.

In the following proposition, we give the best-case HLA factors in the two m-MIMO regimes.

Proposition 3: In a DF relay selection system in the $\mathrm{m}$ MIMO regime, the best-case HLA factor for the $k$-th relay is obtained as

a) m-MIMO I $\left(N_{s} \rightarrow \infty, N_{d}=1\right)$ :

$$
\rho_{\mathrm{k}}^{\mathrm{bc}}= \begin{cases}\frac{1}{2}+\vartheta_{k}, & \text { if } \vartheta_{k} \leq \frac{1}{2}, \\ 1, & \text { if } \vartheta_{k}>\frac{1}{2},\end{cases}
$$

where

$$
\vartheta_{k} \triangleq \frac{\kappa_{r d}^{2}}{2 \kappa_{r, k}^{2}}+\frac{\sigma_{n}^{2}}{2 P_{r} \kappa_{r, k}^{2}\left|h_{r d, k}\right|^{2}} .
$$

b) m-MIMO II $\left(N_{s} \rightarrow \infty, N_{d} \rightarrow \infty\right)$ :

$$
\rho_{k}^{\mathrm{bc}}=\frac{1}{2} \text {. }
$$

Proof: The proof is given in Appendix E.

Armed with this intermediate result, in the next subsection, we provide a practical analysis by averaging over the channel statistics.

\section{B. Evaluation of HWI Levels Over Channel Statistics}

In the m-MIMO I system, the best-case HWI level at each relay is a function of the channel coefficient of the second hop. So the HWI allocation between the transmitter and receiver sides can be addressed by solving:

$$
\begin{aligned}
\gamma_{\text {tot }, k}= & \max _{0<\rho_{k}<1} \mathrm{E}_{\mathrm{h}}\left[\operatorname { m i n } \left\{\frac{1}{\rho_{k}^{2} \kappa_{r, k}^{2}},\right.\right. \\
& \left.\left.\frac{P_{r}\left|h_{r d, k}\right|^{2}}{P_{r}\left(\kappa_{r d}^{2}+\left(1-\rho_{k}\right)^{2} \kappa_{r, k}^{2}\right)\left|h_{r d, k}\right|^{2}+\sigma_{n}^{2}}\right\}\right],
\end{aligned}
$$

where $\mathrm{E}_{\mathrm{h}}[\cdot]$ denotes the expectation over channel coefficients. Using (54) and ignoring the terms independent of $\rho_{k}$, the value of HWI level that maximizes (28) is obtained as

$$
\begin{aligned}
\rho_{k}^{\mathrm{bc}, \text { stoch }}= & \arg \min _{0<\rho_{k}<1}\left(\left(1-\kappa_{2, k}^{2} \gamma_{k}^{\max }\right) e^{-x_{k}^{\max }}\right. \\
& \left.+\varsigma_{k} e^{\varsigma_{k}}\left(E_{1}\left(\varsigma_{k}\right)-E_{1}\left(x_{k}^{\max }+\varsigma_{k}\right)\right)\right) .
\end{aligned}
$$

With $\kappa_{r r, k}=\rho_{k} \kappa_{r, k} \quad$ and $\quad \kappa_{t r, k}=\left(1-\rho_{k}\right) \kappa_{r, k}$, we have $\quad \gamma_{k}^{\max }=\min \left\{\left(\rho_{k}^{2} \kappa_{r, k}^{2}\right)^{-1},\left(\left(1-\rho_{k}\right)^{2} \kappa_{r, k}^{2}+\kappa_{r d}^{2}\right)^{-1}\right\}$, 
$x_{k}^{\max }=\frac{\sigma_{n}^{2} \min \left\{\left(\rho_{k}^{2} \kappa_{r, k}^{2}\right)^{-1},\left(\left(1-\rho_{k}\right)^{2} \kappa_{r, k}^{2}+\kappa_{r d}^{2}\right)^{-1}\right\}}{P_{r}\left(1-\min \left\{\left(\rho_{k}^{2} \kappa_{r, k}^{2}\right)^{-1}\left(\left(1-\rho_{k}\right)^{2} \kappa_{r, k}^{2}+\kappa_{r d}^{2}\right), 1\right\}\right)} \quad$ and $\quad \varsigma_{k}=$ $\frac{\sigma_{n}^{2}}{P_{r}\left(\left(1-\rho_{k}\right)^{2} \kappa_{r, k}^{2}+\kappa_{r d}^{2}\right)}$, which are non-convex functions of optimization variable $\rho_{k}$. These in turn make the HLA problem non-convex. However, according to (25), the HLA factor becomes less dependent on the channel coefficients as SNR increases. Therefore, for high SNRs, we can obtain an approximate solution of the stochastic HLA problem by calculating the expected value of the expression in (25) over the channel coefficients. According to (25), if $\kappa_{r d}>\kappa_{r, k}$, then $\vartheta_{k}$ is always greater than one, so we have $\mathrm{E}_{\mathrm{h}}\left[\rho_{\mathrm{k}}^{\mathrm{bc}}\right]=1$. On the other hand, if $\kappa_{r d} \leq \kappa_{r, k}$, knowing that $\left|h_{r d, k}\right|^{2}$ follows a unit rate exponential distribution and after some calculations, we obtain

$$
\begin{aligned}
\rho_{\mathrm{k}}^{\mathrm{bc}, \text { stoch }} \simeq & \mathrm{E}_{\mathrm{h}}\left[\rho_{\mathrm{k}}^{\mathrm{bc}}\right] \\
= & \left(\frac{1}{2}+\mathrm{E}_{\mathrm{h}}\left[\vartheta_{k} \mid \vartheta_{k} \leq \frac{1}{2}\right]\right) P\left(\vartheta_{k} \leq \frac{1}{2}\right) \\
& +P\left(\vartheta_{k}>\frac{1}{2}\right) \\
= & 1+\left(\frac{\kappa_{r d}^{2}}{2 \kappa_{r, k}^{2}}-\frac{1}{2}\right) e^{-x_{t h}}+\frac{\sigma_{n}^{2}}{2 P_{r} \kappa_{r, k}^{2} x} E_{1}\left(x_{t h}\right)
\end{aligned}
$$

where $x_{t h} \triangleq \frac{\sigma_{n}^{2}}{P_{r}\left(\kappa_{r, k}^{2}-\kappa_{r d}^{2}\right)}$. Combining these results

$$
\rho_{\mathrm{k}}^{\mathrm{bc}, \text { stoch }} \simeq \begin{cases}1+\left(\frac{\kappa_{r d}^{2}}{2 \kappa_{r, k}^{2}}-\frac{1}{2}\right) e^{-x_{t h}} & \text { if } \kappa_{r d} \leq \kappa_{r, k}, \\ +\frac{\sigma_{n}^{2}}{2 P_{r} \kappa_{r, k}^{2} x} E_{1}\left(x_{t h}\right), & \\ 1, & \text { if } \kappa_{r d}>\kappa_{r, k} .\end{cases}
$$

As also observed in the previous sections, this result basically shows that the best HLA is the one which makes the SNDRs of the two hops as close to each other as possible. Since the obtained HWI level allocation is only a function of system parameters such as HWI levels (i.e., it is independent of the instantaneous channel coefficients), it can be utilized in practice as a guideline for an overall system design under a total cost constraint. We also note that the results in Theorem 4 and Proposition 3 serve as upper bounds on the system performance averaged over fading statistics.

\section{SCALABILITY ANALYSIS}

As the number of relays increases, the degrees of freedom and the number of channel coefficients to choose from also increase. Therefore, in the limit of the number of relays tending to infinity, the maximum possible end-to-end SNDR can be achieved for each channel use, i.e.,

$$
\begin{aligned}
\lim _{K \rightarrow \infty} \max _{k \in\{K\}}\left\{\gamma_{\min , k}\right\} & =\sup \left\{\gamma: F_{\Gamma_{\min }}(\gamma)<1\right\} \\
& \triangleq \omega\left(F_{\Gamma_{\min }}\right) \\
& =\min \left\{\left(\kappa_{r r, k}^{2}\right)^{-1},\left(\kappa_{t r, k}^{2}\right)^{-1}\right\} .
\end{aligned}
$$

That is, we know the limiting value of the end-to-end SNDR. However, we are also interested in its limiting characteristics, which is tightly related to the system performance.

Extreme value theory, basically, states that the limiting distribution of the maximum of $K$ i.i.d. random variables with a common $\mathrm{CDF} F_{X}(x)$, if exists, after proper renormalization can only converge in distribution to one of three possible distributions, namely, Gumbel, Fréchet, or Weibull distributions [20], i.e.,

$$
\lim _{K \rightarrow \infty} F_{X}^{K}\left(a_{K} x+b_{K}\right)=G_{X}(x)
$$

where $G_{X}(x)$ is the CDF of Gumbel, Fréchet, or Weibull distributions, and $a_{K}$ and $b_{K}$ are the normalization coefficients.

\section{A. M-MIMO I System}

We first recall a lemma:

Lemma 3 ([19, Theorem 10.5.2], [20, Theorem 3.3]): If there exists an $x_{1}$ that in the open interval $\left(x_{1}, \omega(F)\right)$ probability density function (PDF) of $f_{X}(x)$ is positive and the following holds,

$$
\lim _{x \rightarrow \omega(F)} \frac{d}{d x}\left[\frac{1-F_{X}(x)}{f_{X}(x)}\right]=0,
$$

then, the limiting distribution of maximum of $K$ i.i.d. random variables with $\mathrm{CDF} F_{X}(x)$, after proper normalization, converges in distribution to a Gumbel distribution; i.e., there exist proper normalization coefficients such that

$$
\lim _{K \rightarrow \infty} F_{X}^{K}\left(a_{K} x+b_{K}\right)=\exp \left(-e^{-x}\right),
$$

where the normalization coefficients can be obtained as

$$
b_{K}=F_{X}^{-1}\left(1-\frac{1}{K}\right), \quad a_{K}=F_{X}^{-1}\left(1-\frac{1}{K e}\right)-b_{K},
$$

where $F_{X}^{-1}(\cdot)$ is the inverse function of $F_{X}(\cdot)$ and $e$ is the Napier constant.

Theorem 5: In the m-MIMO I system $\left(N_{s} \rightarrow \infty, N_{d}=1\right)$, assuming that all the relays have the same levels of transmit HWI $\left(\kappa_{t r, k}=\kappa_{t r}, \forall k \in[K]\right.$ ), as the number of relays increases, the maximum of the SNDRs of the second hop converges in distribution to a Gumbel distribution; i.e.,

$$
\lim _{K \rightarrow \infty} F_{\Gamma_{r d, k}}^{K}\left(a_{K} \gamma+b_{K}\right)=\exp \left(-e^{-\gamma}\right),
$$

where the normalization coefficients can be obtained as

$$
a_{K}=\frac{P_{r} \sigma_{n}^{2}}{\left(P_{r} \ln (K) \kappa_{2}^{2}+\sigma_{n}^{2}\right)^{2}}, \quad b_{K}=\frac{P_{r} \ln (K)}{P_{r} \ln (K) \kappa_{2}^{2}+\sigma_{n}^{2}},
$$

where $\kappa_{2} \triangleq \sqrt{\kappa_{t r}^{2}+\kappa_{r d}^{2}}$.

Proof: The proof is given in Appendix F.

\section{B. M-MIMO II System}

According to (14), since the end-to-end SNDR of the mMIMO II is independent of the relay indices, increasing the number of relays has no effect on it. However, that is not the case when there are HWI level variations at the relays. As discussed earlier, in the limit of the number of relays tending to infinity, the maximum possible end-to-end SNDR can be achieved in each realization. So, in the m-MIMO II system with HWI level variations at the relays, the limiting value of the end-to-end 
SNDR as the number of relays increases is equal to

$$
\lim _{K \rightarrow \infty} \gamma_{\text {tot }}^{\mathrm{II}} \stackrel{\text { a.s. }}{\rightarrow}\left(\bar{\kappa}-\frac{\delta_{\kappa}}{2}\right)^{-2}
$$

We are also interested in the limiting characteristics of the end-to-end SNDR. We recall the following result:

Lemma 4 ([19, Theorem 10.5.2], [20, Theorem 3.3]): If $\omega(F)<\infty$ and for some $\alpha>0$ the following holds,

$$
\lim _{x \rightarrow \omega(F)} \frac{(\omega(F)-x) f_{X}(x)}{1-F_{X}(x)}=\alpha,
$$

then, the limiting distribution of maximum of $K$ i.i.d. random variables with $\mathrm{CDF} F_{X}(x)$, after proper normalization, converges in distribution to a Weibull distribution; i.e., there exist proper normalization coefficients such that

$$
\lim _{K \rightarrow \infty} F_{X}^{K}\left(c_{K} x+d_{K}\right)= \begin{cases}1, & \gamma>0 \\ \exp \left(-(-x)^{\alpha}\right), & \gamma \leq 0\end{cases}
$$

where the normalization coefficients can be obtained as

$$
c_{K}=\omega(F)-F_{X}^{-1}\left(1-\frac{1}{K}\right), \quad d_{K}=\omega(F) .
$$

Theorem 6: In the m-MIMO II system $\left(N_{s} \rightarrow \infty, N_{d} \rightarrow \infty\right)$ with DF relay selection and random, uniformly distributed HWI levels at the relays, as the number of relays increases, the end-toend SNDR of the system converges in distribution to a Weibull distribution; i.e.,

$$
\lim _{K \rightarrow \infty}\left(F_{\Gamma_{\min }}^{I I}\left(c_{K} \gamma+d_{K}\right)\right)^{K}= \begin{cases}1, & \gamma>0, \\ \exp \left(-(-\gamma)^{2}\right), & \gamma \leq 0,\end{cases}
$$

where the normalization coefficients are as follows

$$
\begin{aligned}
& c_{K}=\left(\bar{\kappa}-\frac{\delta_{\kappa}}{2}\right)^{-2}-\left(\bar{\kappa}+\delta_{\kappa}\left(\frac{1}{\sqrt{K}}-\frac{1}{2}\right)\right)^{-2}, \\
& d_{K}=\left(\bar{\kappa}-\frac{\delta_{\kappa}}{2}\right)^{-2} .
\end{aligned}
$$

Proof: The proof is given in Appendix F.

\section{Simulations AND Numerical Results}

In this section, we present several simulation results and numerical evaluations to corroborate our findings and to study the system performance. All the simulations are obtained by averaging 1000 realizations of the system. The default values of the parameters used in the simulations are as follows:

$$
\begin{aligned}
& P_{s}=1 \mathrm{~W}, \quad \mathrm{P}_{\mathrm{r}}=1 \mathrm{~W}, \quad \sigma_{\mathrm{n}}^{2}=1, \quad \kappa_{\mathrm{ts}}=0.1, \\
& \kappa_{r r}=0.12, \quad \kappa_{t r}=0.13, \quad \kappa_{r d}=0.11,
\end{aligned}
$$

where the HWI levels are chosen in the range [0.08, 0.175] in accordance with the error vector magnitudes of 3GPP LTE requirements [30].

The end-to-end SNDR of the system versus the number of end-node antennas (assuming $N_{s}=N_{r}=N$ ) for different numbers of relay nodes are presented in Fig. 2, assuming that the relay nodes have similar levels of HWI. According to Fig. 2, as the number of end-node antennas increases, the end-to-end SNDR converges to the theoretically attained m-MIMO expression for all numbers of relay nodes, as expected. Also, for moderate number of end-node antennas, the end-to-end SNDR

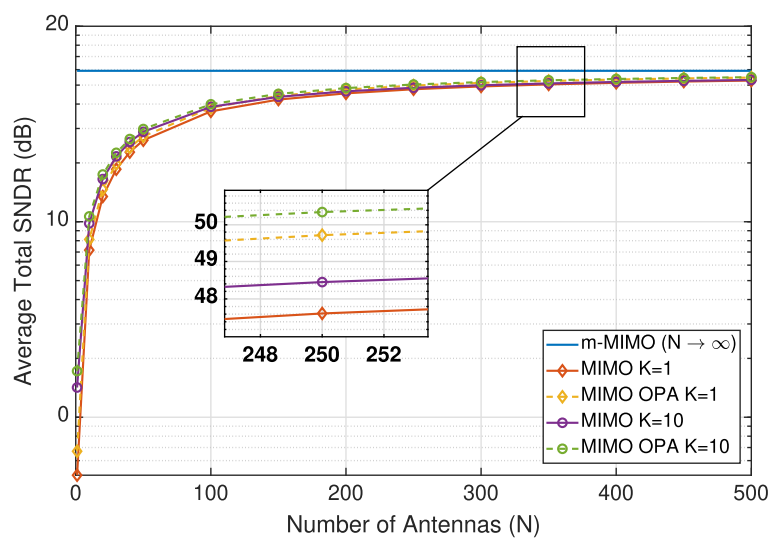

Fig. 2. Average end-to-end SNDR versus number of antennas.

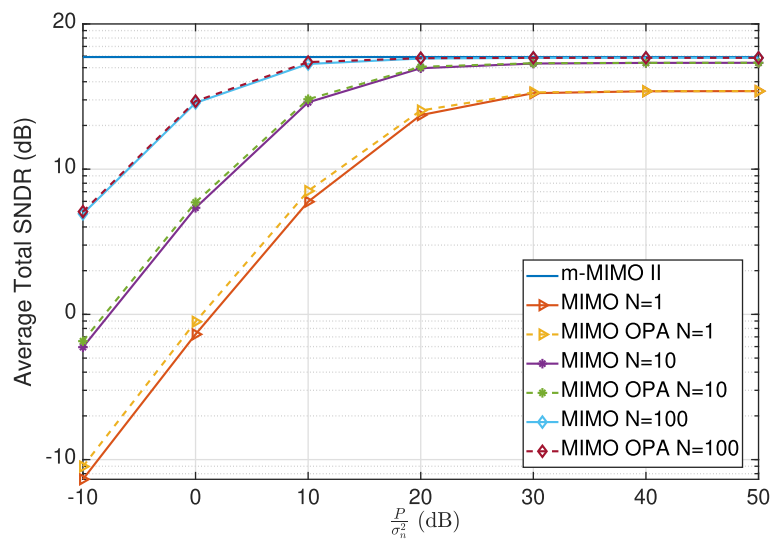

Fig. 3. Average total SNDR versus total transmit SNR for $K=10$.

of the system increases as the number of relay nodes increases. However, in the m-MIMO II regime (high numbers of end-node antennas), the end-to-end SNDR is almost unaffected by increasing the number of relay nodes. For instance, all diagrams achieve SNDRs of around $13 \mathrm{~dB}$ when $N=100$, however, SNDRs increase only about $1 \mathrm{~dB}$ by doubling the number of antennas.

In Fig. 3, average end-to-end SNDR versus total transmit signal-to-noise ratio (SNR) $\left(\frac{P}{\sigma_{n}^{2}}=\frac{P_{s}+P_{r}}{\sigma_{n}^{2}}\right)$ is presented. According to Fig. 3, as the total transmit SNR increases, the average end-to-end SNDR increases as well until reaching the interference-limited regime, where it saturates. It can also be seen that the improvement due to power allocation diminishes as the number of antennas increases.

Average end-to-end SNDR versus the number of relay nodes is plotted in Fig. 4. It is observed that, as the number of relay nodes increases, the average end-to-end SNDR increases as well. This increase is less pronounced as the number of antennas increases, to the extent that, in the m-MIMO II regime, the system performance becomes unaffected by the number of relay nodes. We can also see that the improvement due to power allocation mostly depends on the number of antennas rather than the number of relay nodes.

The effects of HWI on the system performance is investigated in Fig. 5 for different numbers of relay nodes and $K=10$. We assume that the source and the destination have the same 


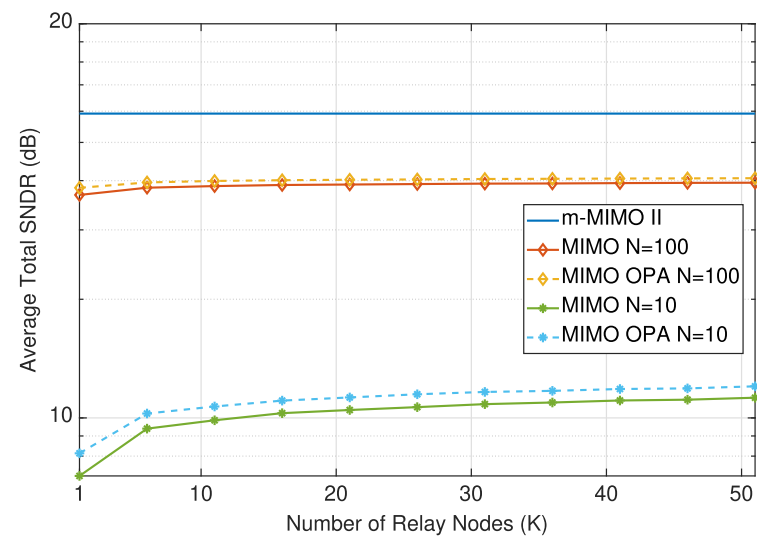

Fig. 4. Average end-to-end SNDR versus number relay nodes.

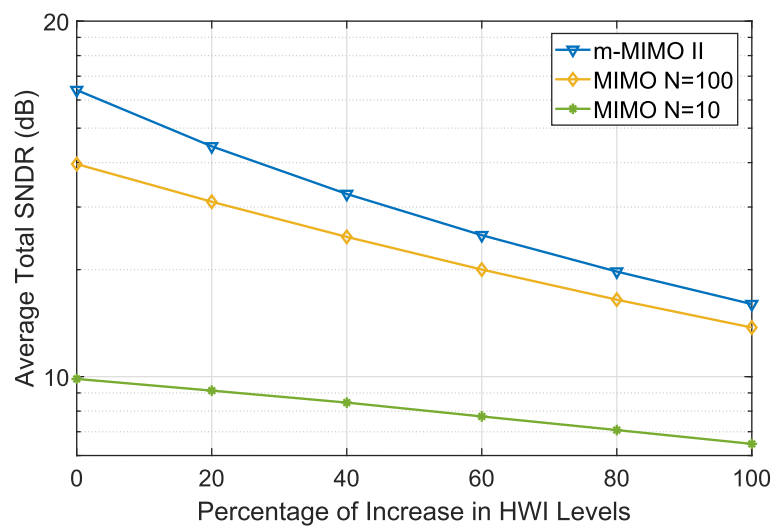

Fig. 5. Average end-to-end SNDR versus percentage of increase in HWI levels (compared to the default values) for $K=10$.

number of antennas. The end-to-end SNDR of the system versus percentage of increase in HWI levels, compared to the default values of (45), is presented in Fig. 5, which shows that as the impairment levels increase the end-to-end SNDR decreases. We also observe that the system performance is more sensitive to HWI levels in the m-MIMO II regime. This is due to the fact that in the m-MIMO II regime, the system performance depends mostly on HWI levels rather than the other parameters, which can be averaged over. For instance, the SNDR reduces about $2 \mathrm{~dB}$ by $50 \%$ increase in HWI levels for $N=10$, while this reduction is around $4 \mathrm{~dB}$ in the m-MIMO II regime.

In Fig. 6, the effects of variations of HWI levels among relays on the average end-to-end SNDR is analyzed for the m-MIMO II system for $\bar{\kappa}=0.1$ and different values of $K$. According to Fig. 6, increasing the number of relays and the HWI level variations among relays increases the average end-to-end SNDR of the system as well. This is due to the fact that increasing these parameters increases the chance of having a good relay (with a low HWI level) among all available relays; hence, improving the system performance. It should also be noted that the average end-to-end SNDR is not an increasing function of $\delta_{\kappa}$ in general. Also, our proposed lower bound is tight even in higher values of $\frac{\delta_{\kappa}}{\bar{\kappa}}$, e.g., a gap of only $0.5 \%$ for $K=20$ in $\frac{\delta_{K}}{\bar{\kappa}}=0.3$.

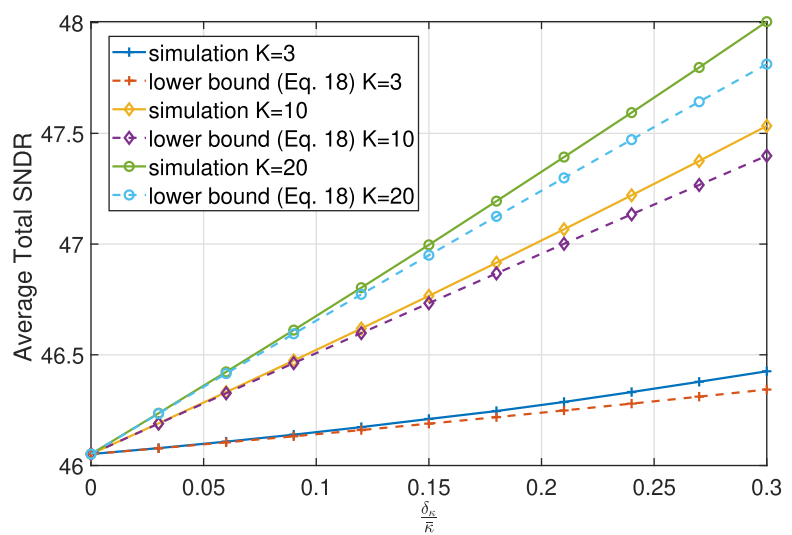

Fig. 6. Average end-to-end SNDR versus normalized range of HWI level variations at the relays of the m-MIMO II system for $\bar{\kappa}=0.1$.

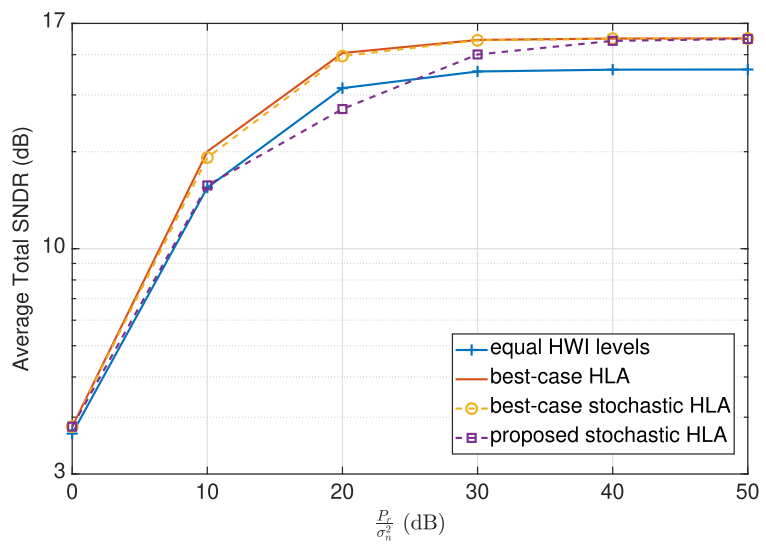

Fig. 7. Comparison of stochastic and non-stochastic approaches of HWI level allocation for $\kappa_{r}=0.25$ and $K=10$

In Fig. 7, the performances of different HWI level allocation algorithms are investigated for m-MIMO I as transmit SNR at the relays $\left(\frac{P_{r}}{\sigma_{n}^{2}}\right)$ increases, where "equal HWI levels" refers to $\kappa_{r r}=\kappa_{t r}=0.5 \kappa_{r}$. In this setup, we assume $K=10$ and $\kappa_{r}=0.25$. According to Fig. 7, employing suitable HWI level allocation improves the system performance from about $5 \%$ for $\mathrm{SNR}=0 \mathrm{~dB}$ up to about $25 \%$ for $\mathrm{SNR}=30 \mathrm{~dB}$. Also, the proposed stochastic HWI level allocation algorithm has almost the same performance as the best-case non-stochastic HLA algorithm (an upper bound on the performance of the stochastic approach) in higher SNRs.

\section{CONCLUSION}

In this paper, considering a cooperative m-MIMO system with multiple DF relays and employing optimal relay selection, we analyze the system performance in the presence of HWI for two m-MIMO scenarios: massive-antenna source and single-antenna destination, and massive-antenna source and destination. We investigate the scalability of the system, by showing that by increasing the number of relays, the normalized end-to-end SNDR tends to a Gumbel distribution for the m-MIMO I system, while it converges to a Weibull distribution for the m-MIMO 
II system with HWI level variations among the relays. We also provide closed form expressions for the optimal power allocation between the source and the selected relay, and the effects of HWI level distributions between the receiving and the transmitting parts of the relay. It is shown that the optimal distribution of the power is the one which makes the SNDRs of the two hops as close to each other as possible. We also find that increasing the number of relays increases the end-to-end SNDR of the $\mathrm{m}$-MIMO systems as well, and the increase is more pronounced when there are variations in the HWI levels among relays. Analysis of the effects of imperfect CSI on system performance with HWI is an interesting possible future direction.

\section{APPENDIX A \\ PROOF OF PROPOSITION 1}

In the first hop, since the source is equipped with massive number of antennas, for the $k$-th relay we have $\gamma_{s r, k}^{\mathrm{m}-\mathrm{MIMO}}=$ $\lim _{N_{s} \rightarrow \infty} \gamma_{s r, k} \stackrel{a . s .}{\rightarrow} \frac{1}{\kappa_{r r, k}^{2}}$. So the CDF of SNDR at the first hop becomes

$$
F_{\Gamma_{s r, k}}(\gamma)=u\left(\gamma-\frac{1}{\kappa_{r r, k}^{2}}\right) .
$$

Since $h_{r d, k} \sim C N(0,1)$, then $\left|h_{r d, k}\right|^{2}$ follows a unit rate exponential distribution; i.e., $\left|h_{r d, k}\right|^{2} \sim \operatorname{Exp}(1)$. Hence, using (7), the CDF of SNDR at the first hop for $0 \leq \gamma \leq\left(\kappa_{t r, k}^{2}+\kappa_{r d}^{2}\right)^{-1}$ is obtained as follows:

$$
\begin{aligned}
F_{\Gamma_{r d, k}}(\gamma) & =P\left(\left|h_{r d, k}\right|^{2} \leq \frac{\sigma_{n}^{2} \gamma}{P_{r}\left(1-\left(\kappa_{t r, k}^{2}+\kappa_{r d}^{2}\right) \gamma\right)}\right) \\
& =1-\exp \left(\frac{-\sigma_{n}^{2} \gamma}{P_{r}\left(1-\left(\kappa_{t r, k}^{2}+\kappa_{r d}^{2}\right) \gamma\right)}\right) .
\end{aligned}
$$

Since the channel coefficients of the two hops are independent of each other, using (9) and after some calculations, the CDF of the minimum SNDR of two hops for each relay, $F_{\Gamma_{\min , k}}(\gamma)$, for $0 \leq \gamma$ is obtained as

$$
\begin{aligned}
F_{\Gamma_{\min , k}}(\gamma) & =1-P\left(\gamma_{s r, k} \geq \gamma\right) P\left(\gamma_{r d, k} \geq \gamma\right) \\
& =1-\exp \left(\frac{-\sigma_{n}^{2} \gamma}{P_{r}\left(1-\kappa_{2, k}^{2} \gamma\right)}\right) u\left(\gamma_{k}^{\max }-\gamma\right) .
\end{aligned}
$$

Finally, since the channel coefficients of the relays are independent of each other, using (48), the CDF of the end-to-end SNDR of the system, $F_{\Gamma_{\max }}(\gamma)$, can be obtained as

$$
\begin{aligned}
F_{\Gamma_{\max }}(\gamma) & =P\left(\max _{k \in\{K\}}\left\{\gamma_{\min , k}\right\} \leq \gamma\right) \\
& =\prod_{k=1}^{K} F_{\Gamma_{\min , k}}(\gamma),
\end{aligned}
$$

which concludes the proof.

\section{APPENDIX B \\ PROOF OF THEOREM 1}

Since $\max (\cdot)$ is a convex function, using Jensen's inequality, we have

$$
\begin{aligned}
\bar{\gamma}_{t o t} & =\mathrm{E}_{\mathrm{h}}\left[\max _{k \in[K]} \min \left\{\gamma_{s r, k}, \gamma_{r d, k}\right\}\right] \\
& \geq \max _{k \in[K]} \mathrm{E}_{\mathrm{h}}\left[\min \left\{\gamma_{s r, k}, \gamma_{r d, k}\right\}\right] .
\end{aligned}
$$

Using (48), we have

$$
\begin{aligned}
f_{\Gamma_{\min , k}}(\gamma)= & \frac{\sigma_{n}^{2} \exp \left(\frac{-\sigma_{n}^{2} \gamma}{P_{r}\left(1-\kappa_{2, k}^{2} \gamma\right)}\right)}{P_{r}\left(1-\kappa_{2, k}^{2} \gamma\right)^{2}} u\left(\gamma_{k}^{\max }-\gamma\right) \\
& +\exp \left(\frac{-\sigma_{n}^{2} \gamma_{k}^{\max }}{P_{r}\left(1-\kappa_{2, k}^{2} \gamma_{k}^{\max }\right)}\right) \delta\left(\gamma-\gamma_{k}^{\max }\right),
\end{aligned}
$$

where $\delta(\cdot)$ denotes Dirac delta function. The expected value in (25) can be written as

$$
\begin{aligned}
\mathrm{E}_{\mathrm{h}}\left[\Gamma_{\min , k}\right]= & \int_{0}^{\gamma_{k}^{\max }} \frac{\sigma_{n}^{2} \gamma \exp \left(\frac{-\sigma_{n}^{2} \gamma}{P_{r}\left(1-\kappa_{2, k}^{2} \gamma\right)}\right)}{P_{r}\left(1-\kappa_{2, k}^{2} \gamma\right)^{2}} d \gamma \\
& +\gamma_{k}^{\max } \exp \left(\frac{-\sigma_{n}^{2} \gamma_{k}^{\max }}{P_{r}\left(1-\kappa_{2, k}^{2} \gamma_{k}^{\max }\right)}\right)
\end{aligned}
$$

With a change of variable $x=\frac{\sigma_{n}^{2} \gamma}{P_{r}\left(1-\kappa_{2, k}^{2} \gamma\right)}$, the first integral in (52) turns into

$$
\begin{aligned}
& \int_{0}^{x_{k}^{\max }} \frac{P_{r} x e^{-x}}{\sigma_{n}^{2}+\kappa_{2, k}^{2} P_{r} x} d x \\
& =\frac{1}{\kappa_{2, k}^{2}} \int_{0}^{x_{k}^{\max }} e^{-x} d x-\frac{\sigma_{n}^{2}}{\kappa_{2, k}^{2}} \int_{0}^{x_{k}^{\max }} \frac{1}{\sigma_{n}^{2}+\kappa_{2, k}^{2} P_{r} x} e^{-x} d x \\
& =\frac{1}{\kappa_{2, k}^{2}}\left(1-e^{-x_{k}^{\max }}\right)-\frac{\sigma_{n}^{2}}{\kappa_{2, k}^{4} P_{r}} e^{\frac{\sigma_{n}^{2}}{\kappa_{2, k}^{2} P_{r}}} \int_{\frac{\sigma_{n}^{2}}{\kappa_{2, k}^{2} P_{r}}}^{x_{n}^{\max }+\frac{\sigma_{n}^{2}}{\kappa_{2, k}^{2} P_{r}}} \frac{e^{-z}}{z} d z \\
& =\frac{1}{\kappa_{2, k}^{2}}\left(1-e^{-x_{k}^{\max }}-\varsigma_{k} e^{\varsigma_{k}}\left(E_{1}\left(\varsigma_{k}\right)-E_{1}\left(x_{k}^{\max }+\varsigma_{k}\right)\right)\right) .
\end{aligned}
$$

Substituting (53) into (52), we have

$$
\begin{aligned}
\mathrm{E}_{\mathrm{h}}\left[\Gamma_{\min , k}\right]= & \frac{1}{\kappa_{2, k}^{2}}\left(1-\left(1-\kappa_{2, k}^{2} \gamma_{k}^{\max }\right) e^{-x_{k}^{\max }}\right. \\
& \left.-\varsigma_{k} e^{\varsigma_{k}}\left(E_{1}\left(\varsigma_{k}\right)-E_{1}\left(x_{k}^{\max }+\varsigma_{k}\right)\right)\right) .
\end{aligned}
$$

Finally, substituting (54) into (50) concludes the proof. 
APPENDIX C

PROOF OF THEOREM 2

Let $\varepsilon_{r r, k}, \varepsilon_{t r, k} \sim \mathcal{U}\left(\frac{-1}{2}, \frac{1}{2}\right)$, then we have $\kappa_{r r, k}=\bar{\kappa}+$ $\delta_{\kappa} \varepsilon_{r r, k}$ and $\kappa_{t r, k}=\bar{\kappa}+\delta_{\kappa} \varepsilon_{t r, k}$. So, for $\delta_{\kappa} \ll \bar{\kappa}$, we have

$$
\begin{aligned}
\bar{\gamma}_{t o t}^{I I} & =\mathrm{E}\left[\max _{k \in[K]} \min \left\{\frac{1}{\kappa_{r r, k}^{2}}, \frac{1}{\kappa_{t r, k}^{2}}\right\}\right] \\
& =\mathrm{E}\left[\left(\max _{k \in[K]} \min \left\{\frac{1}{\bar{\kappa}+\delta_{\kappa} \varepsilon_{r r, k}}, \frac{1}{\bar{\kappa}+\delta_{\kappa} \varepsilon_{t r, k}}\right\}\right)^{2}\right] \\
& \geq \mathrm{E}\left[\left(\max _{k \in[K]} \min \left\{\frac{1}{\bar{\kappa}}-\frac{\delta_{\kappa}}{\bar{\kappa}^{2}} \varepsilon_{r r, k}, \frac{1}{\bar{\kappa}}-\frac{\delta_{\kappa}}{\bar{\kappa}^{2}} \varepsilon_{t r, k}\right\}\right)^{2}\right] \\
& =\mathrm{E}\left[\left(\frac{1}{\bar{\kappa}}-\frac{\delta_{\kappa}}{\bar{\kappa}^{2}} \min _{k \in[K]} \max \left\{\varepsilon_{r r, k}, \varepsilon_{r r, k}\right\}\right)^{2}\right] \\
& =\frac{1}{\bar{\kappa}^{2}}-\frac{2 \delta_{\kappa}}{\bar{\kappa}^{3}} \mathrm{E}_{1}+\frac{\delta_{\kappa}^{2}}{\bar{\kappa}^{4}} \mathrm{E}_{2},
\end{aligned}
$$

where

$$
\begin{aligned}
& \mathrm{E}_{1} \triangleq \mathrm{E}\left[\min _{\mathrm{k} \in[\mathrm{K}]} \max \left\{\varepsilon_{\mathrm{rr}, \mathrm{k}}, \varepsilon_{\mathrm{rr}, \mathrm{k}}\right\}\right] \\
& \mathrm{E}_{2} \triangleq \mathrm{E}\left[\left(\min _{\mathrm{k} \in[\mathrm{K}]} \max \left\{\varepsilon_{\mathrm{rr}, \mathrm{k}}, \varepsilon_{\mathrm{rr}, \mathrm{k}}\right\}\right)^{2}\right] .
\end{aligned}
$$

Since the CDFs of $\varepsilon_{r r, k}$ and $\varepsilon_{t r, k}$ are the same and equal to $F_{E_{r r}}(\varepsilon)=F_{E_{t r}}(\varepsilon)=\varepsilon+\frac{1}{2}$ in the interval $\left[\frac{-1}{2}, \frac{1}{2}\right]$, we have

$$
\begin{aligned}
F_{E \min }(\varepsilon) & =P\left(\min _{k \in[K]} \max \left\{\varepsilon_{r r, k}, \varepsilon_{r r, k}\right\} \leq \varepsilon\right) \\
& =1-\left(1-\left(\varepsilon+\frac{1}{2}\right)^{2}\right)^{K} .
\end{aligned}
$$

Using binomial expansion $\left((1-\omega)^{K-1}=\sum_{k=0}^{K-1}\left(\begin{array}{c}K-1 \\ k\end{array}\right)(-\omega)^{k}\right)$, we have

$$
\begin{aligned}
I_{K}(\alpha) & \triangleq \int_{0}^{1} \omega^{\alpha}(1-\omega)^{K-1} d \omega \\
& =\sum_{k=0}^{K-1}\left(\begin{array}{c}
K-1 \\
k
\end{array}\right)(-1)^{k} \frac{1}{k+\alpha+1} .
\end{aligned}
$$

So, using (56) and (57) and a change of variable $\left(\omega=\left(\varepsilon+\frac{1}{2}\right)^{2}\right)$, the values of the first and the second expectations in (55) become

$$
\begin{aligned}
\mathrm{E}_{1} & =\mathrm{K} \int_{0}^{1}\left(\sqrt{\omega}-\frac{1}{2}\right)(1-\omega)^{\mathrm{K}-1} \mathrm{~d} \omega=\mathrm{KI}_{\mathrm{K}}\left(\frac{1}{2}\right)-\frac{1}{2} \\
\mathrm{E}_{2} & =K \int_{0}^{1}\left(\sqrt{\omega}-\frac{1}{2}\right)^{2}(1-\omega)^{K-1} d \omega \\
& =K\left(I_{K}(1)-I_{K}\left(\frac{1}{2}\right)\right)+\frac{1}{4}
\end{aligned}
$$

respectively. Substituting (58) and (59) in (55) gives us (16).

\section{APPENDIX D \\ PROOFS OF THEOREM 3 AND PROPOSITION 2}

\section{A. Proof of Theorem 3}

The optimal power allocation factor of the $k$-th relay can be obtained by solving the optimization problem in (17). It can easily be shown that, in the interval $0<\lambda_{k}<1$, the expression $\frac{\lambda_{k} P\left\|\mathbf{h}_{s r, k}\right\|^{2}}{\lambda_{k} P \alpha_{s r, k}^{2}\left\|\mathbf{h}_{s r, k}\right\|^{2}+\sigma_{n}^{2}}$ is an increasing function of $\lambda_{k}$ starting from a zero value while the expression $\frac{\left(1-\lambda_{k}\right) P\left\|\mathbf{h}_{r d, k}\right\|^{2}}{\left(1-\lambda_{k}\right) P \alpha_{r d, k}^{2}\left\|\mathbf{h}_{r d, k}\right\|^{2}+\sigma_{n}^{2}}$ is a decreasing function ending in a zero value. Hence, they intersect once in the interval. Since one function is increasing and the other decreasing and they intersect, the maximum value of their minimum is achieved at the intersection point. In other words, for the optimal power allocation factor, we have

$$
\frac{\lambda_{k}^{\mathrm{opt}} P\left\|\mathbf{h}_{s r, k}\right\|^{2}}{\lambda_{k}^{\mathrm{opt}} P \alpha_{s r, k}^{2}\left\|\mathbf{h}_{s r, k}\right\|^{2}+\sigma_{n}^{2}}=\frac{\left(1-\lambda_{k}^{\mathrm{opt}}\right) P\left\|\mathbf{h}_{r d, k}\right\|^{2}}{\left(1-\lambda_{k}^{\mathrm{opt}}\right) P \alpha_{r d, k}^{2}\left\|\mathbf{h}_{r d, k}\right\|^{2}+\sigma_{n}^{2}} .
$$

The Eq. (60) can be turned into a quadratic equation of the form $a_{k}^{\prime}\left(\lambda_{k}^{\text {opt }}\right)^{2}+b_{k}^{\prime} \lambda_{k}^{\text {opt }}+c_{k}^{\prime}=0$ with the following two solutions

$$
\lambda_{k}^{\mathrm{opt}}=\frac{-b_{k}^{\prime} \pm \sqrt{b_{k}^{\prime 2}-4 a_{k}^{\prime} c_{k}^{\prime}}}{2 a_{k}^{\prime}}
$$

where its coefficients are given in (20).

We now analyze which of the two possible solutions in (61) is the optimal one. If $a_{k}^{\prime}$ is negative, since $c_{k}^{\prime}$ is always negative, their ratio $\frac{c_{k}^{\prime}}{a_{k}^{\prime}}$ becomes positive, so the solutions of the quadratic equation have the same signs. Since we previously showed that there is a single solution in the interval $(0,1)$, the other solution is greater than or equal to one. So the optimal solution is the smaller one, which (with $a_{k}^{\prime}$ being negative) corresponds to $\lambda_{k}^{\text {opt }}=\frac{-b_{k}^{\prime}+\sqrt{b_{k}^{\prime 2}-4 a_{k}^{\prime} c_{k}^{\prime}}}{2 a_{k}^{\prime}}$. On the other hand, if $a_{k}^{\prime}$ is positive, the ratio $\frac{c_{k}^{\prime}}{a_{k}^{\prime}}$ becomes negative, hence the solutions of the quadratic equation have different signs, i.e., the other solution is less than or equal to zero. In other words, the optimal solution (the solution in the interval $(0,1))$ becomes the larger root, which in this case ( $a_{k}^{\prime}$ being positive) corresponds again to $\lambda_{k}^{\text {opt }}=\frac{-b_{k}^{\prime}+\sqrt{b_{k}^{\prime}{ }^{2}-4 a_{k}^{\prime} c_{k}^{\prime}}}{2 a_{k}^{\prime}}$. Therefore we can conclude that the optimal power allocation factor can be written as in (19).

\section{B. Proof of Proposition 2}

According to (14), since in the m-MIMO I regime the SNDR is independent of the transmit power, the optimal strategy for the source is to send with the lowest possible power, i.e., $P_{s}^{\text {opt }} \rightarrow$ 0 . Hence, the relay has a maximum power of $P$ available for transmitting its signal, i.e., $0<P_{r}<P$. Now maximizing the end-to-end SNDR, $\min \left\{\frac{1}{\kappa_{r r, k}^{2}}, \frac{P_{r}\left|h_{r d, k}\right|^{2}}{P_{r} \alpha_{r d, k}^{2}\left|h_{r d, k}\right|^{2}+\sigma_{n}^{2}}\right\}$, over $P_{r}$ gives us the optimal transmit power of the relay. If $\frac{1}{\kappa_{r r, k}^{2}}>$ $\max _{P_{r}}\left\{\frac{P_{r}\left|h_{r d, k}\right|^{2}}{P_{r} \alpha_{r d, k}^{2}\left|h_{r d, k}\right|^{2}+\sigma_{n}^{2}}\right\}=\frac{P\left|h_{r d, k}\right|^{2}}{P \alpha_{r d, k}^{2}\left|h_{r d, k}\right|^{2}+\sigma_{n}^{2}}$, then the maximum possible power value maximizes the end-to-end SNDR. Otherwise, any $P_{r}<P$ greater than the intersection of the SNDRs of the two hops maximizes the end-to-end SNDR, 
among which the intersection itself is the total power minimizing point. Solving for the intersection and summing up the results give us (21).

\section{APPENDIX E}

ProOFs OF THEOREM 4 AND PROPOSITION 3

\section{A. Proof of Theorem 4}

It is easy to show that $f_{1}\left(\rho_{k}\right) \triangleq \frac{P_{s}\left\|\mathbf{h}_{s r, k}\right\|^{2}}{P_{s}\left(\beta_{s r, k}+\rho_{k}^{2} \kappa_{r, k}^{2}\left\|\mathbf{h}_{s r, k}\right\|^{2}\right)+\sigma_{n}^{2}}$ is a decreasing function of $\rho_{k}$ in the interval $[0,1]$, while $f_{2}\left(\rho_{k}\right) \triangleq \frac{P_{r}\left\|\mathbf{h}_{r d, k}\right\|^{2}}{P_{r}\left(\beta_{r d, k}+\left(1-\rho_{k}\right)^{2} \kappa_{r, k}^{2}\left\|\mathbf{h}_{r d, k}\right\|^{2}\right)+\sigma_{n}^{2}}$ is an increasing one. So three cases can occur. First, if $f_{1}\left(\rho_{k}\right) \geq f_{2}\left(\rho_{k}\right)$ in the interval $[0,1]$ (corresponding to $\min _{\rho_{k}}\left\{f_{1}\left(\rho_{k}\right)\right\} \geq$ $\max _{\rho_{k}}\left\{f_{2}\left(\rho_{k}\right)\right\}$ or equivalently $\varpi_{k}>2 \kappa_{r}^{2}$ with $\varpi_{k}$ given in (24)), then $\rho_{k}^{\text {bc }}=\arg \max _{\rho_{k}}\left\{f_{2}\left(\rho_{k}\right)\right\}=1$. Secondly, if $f_{1}\left(\rho_{k}\right) \leq f_{2}\left(\rho_{k}\right)$ in the interval $[0,1]$ (corresponding to $\max _{\rho_{k}}\left\{f_{1}\left(\rho_{k}\right)\right\} \leq \min _{\rho_{k}}\left\{f_{2}\left(\rho_{k}\right)\right\}$ or equivalently $\left.\varpi_{k} \leq 0\right)$, then $\rho_{k}^{\mathrm{bc}}=\arg \max _{\rho_{k}}\left\{f_{1}\left(\rho_{k}\right)\right\}=0$. Thirdly, if the two functions intersect in the interval $[0,1]$ (corresponding to $\left.0<\varpi_{k}<2 \kappa_{r}^{2}\right)$, then $\max _{\rho_{k}} \min \left\{f_{1}\left(\rho_{k}\right), f_{2}\left(\rho_{k}\right)\right\}$ is achieved in their intersection. That is, in this case, the best-case HLA factor is obtained by solving

$$
\begin{aligned}
& \frac{P_{s}\left\|\mathbf{h}_{s r, k}\right\|^{2}}{P_{s}\left(\beta_{s r, k}+\left(\rho_{k}^{\mathrm{bc}}\right)^{2} \kappa_{r, k}^{2}\right)\left\|\mathbf{h}_{s r, k}\right\|^{2}+\sigma_{n}^{2}} \\
& =\frac{P_{r}\left\|\mathbf{h}_{r d, k}\right\|^{2}}{P_{r}\left(\beta_{r d, k}+\left(1-\rho_{k}^{\mathrm{bc}}\right)^{2} \kappa_{r, k}^{2}\right)\left\|\mathbf{h}_{r d, k}\right\|^{2}+\sigma_{n}^{2}},
\end{aligned}
$$

which results in $\rho_{k}^{\mathrm{bc}}=\frac{\varpi_{k}}{2 \kappa_{r}^{2}}$, concluding the proof.

\section{B. Proof of Proposition 3}

For the m-MIMO I system, using (14), the allocation problem in (22) turns into

$$
\begin{aligned}
& \gamma_{\text {tot }, k}=\max _{0<\rho_{k}<1} \min \\
& \times\left\{\frac{P_{r}\left|h_{r d, k}\right|^{2}}{P_{r}\left(\kappa_{r d}^{2}+\left(1-\rho_{k}\right)^{2} \kappa_{r, k}^{2}\right)\left|h_{r d, k}\right|^{2}+\sigma_{n}^{2}}, \frac{1}{\rho_{k}^{2} \kappa_{r, k}^{2}}\right\} .
\end{aligned}
$$

Since $f_{1}=\frac{1}{\rho_{k}^{2} \kappa_{r, k}^{2}}$ is a decreasing function of $\rho_{k}$, while $f_{2}=\frac{P_{r}\left|h_{r d, k}\right|^{2}}{P_{r}\left(\kappa_{r d}^{2}+\left(1-\rho_{k}\right)^{2} \kappa_{r, k}^{2}\right)\left|h_{r d, k}\right|^{2}+\sigma_{n}^{2}}$ is an increasing one, two cases can occur. First, if $f_{1}\left(\rho_{k}\right)>f_{2}\left(\rho_{k}\right)$ in the interval $[0,1]$ (corresponding to $\min _{\rho_{k}}\left\{f_{1}\left(\rho_{k}\right)\right\} \geq \max _{\rho_{k}}\left\{f_{2}\left(\rho_{k}\right)\right\}$ or equivalently $\vartheta_{k}>\frac{1}{2}$ with $\vartheta_{k}$ given in (25)), then $\rho_{k}^{\text {bc }}=$ $\arg \max _{\rho_{k}}\left\{f_{2}\left(\rho_{k}\right)\right\}=1$. Secondly, if the two functions intersect in the interval $[0,1]$ (corresponding to $\vartheta_{k} \leq \frac{1}{2}$ ), then $\max _{\rho_{k}} \min \left\{f_{1}\left(\rho_{k}\right), f_{2}\left(\rho_{k}\right)\right\}$ is achieved in their intersection. Solving for the intersection and summing up the above results give us (25).
For the m-MIMO II system, using (14), the allocation problem (22) turns into

$$
\gamma_{\mathrm{tot}, k}=\max _{0<\rho_{k}<1} \min \left\{\frac{1}{\rho_{k}^{2} \kappa_{r, k}^{2}}, \frac{1}{\left(1-\rho_{k}\right)^{2} \kappa_{r, k}^{2}}\right\},
$$

whose solution is attained when the SNDRs of the two hops are equal, i.e., $\rho_{k}^{\mathrm{bc}}=\frac{1}{2}$.

\section{APPENDIX F}

\section{PROOFS OF THEOREM 5 AND THEOREM 6}

\section{A. Proof of Theorem 5}

Using (47) and assuming that all the relays have the same levels of transmit HWI, the PDF of SNDR of each relay in the second hop, $f_{\Gamma_{r d}}(\gamma)$, becomes

$$
f_{\Gamma_{r d}}(\gamma)=\frac{d}{d \gamma} F_{\Gamma_{r d}}(\gamma)=\frac{\sigma_{n}^{2} \exp \left(\frac{-\sigma_{n}^{2} \gamma}{P_{r}\left(1-\kappa_{2}^{2} \gamma\right)}\right)}{P_{r}\left(1-\kappa_{2}^{2} \gamma\right)^{2}} .
$$

Using (65) and (47), we obtain

$$
\lim _{\gamma \rightarrow \omega(F)} \frac{d}{d \gamma} \frac{1-F_{\Gamma_{r d}}(\gamma)}{f_{\Gamma_{r d}}(\gamma)}=\lim _{\gamma \rightarrow \kappa_{2}^{-} 2} \frac{-2 P_{r}}{\sigma_{n}^{2}} \kappa_{2}^{2}\left(1-\kappa_{2}^{2} \gamma\right)=0 .
$$

According to (65), $f_{\Gamma_{r d}}(\gamma)$ is non-negative in the interval $\left(0,\left(\kappa_{t r}^{2}+\kappa_{r d}^{2}\right)^{-1}\right)$. Combining this with the results of Lemma 3 and (66), proof of (37) is completed.

Next, using (47) and after some algebra, the inverse of $F_{\Gamma_{r d}}(\gamma)=q$, becomes

$$
F_{\Gamma_{r d}}^{-1}(q)=\frac{P_{r} \ln (1-q)}{P_{r} \ln (1-q) \kappa_{2}^{2}-\sigma_{n}^{2}} \geq 0 .
$$

Now, using Lemma 3, the normalization coefficients are obtained as in (38).

\section{B. Proof of Theorem 6}

Using (15) and utilizing L'Hôpital's rule, we obtain

$$
\begin{aligned}
& \lim _{\gamma \rightarrow\left(\bar{\kappa}-\frac{\delta_{\kappa}}{2}\right)^{-2}} \frac{\left(\left(\bar{\kappa}-\frac{\delta_{\kappa}}{2}\right)^{-2}-\gamma\right) f_{\Gamma_{\min }}(\gamma)}{1-F_{\Gamma_{\min }}(\gamma)} \\
& =\lim _{\gamma \rightarrow\left(\bar{\kappa}-\frac{\delta_{\kappa}}{2}\right)^{-2}} \frac{\left(\bar{\kappa}-\frac{\delta_{\kappa}}{2}\right)^{-2}-\gamma}{\gamma-\left(\bar{\kappa}-\frac{\delta_{\kappa}}{2}\right) \sqrt{\gamma^{3}}} \\
& \equiv \lim _{\gamma \rightarrow\left(\bar{\kappa}-\frac{\delta_{\kappa}}{2}\right)^{-2}} \frac{-1}{1-\frac{3}{2}\left(\bar{\kappa}-\frac{\delta_{\kappa}}{2}\right) \sqrt{\gamma}} \\
& =2 .
\end{aligned}
$$

Since $\omega(F)=\left(\bar{\kappa}-\frac{\delta_{\kappa}}{2}\right)^{-2}$, by employing Lemma 4 and (68), (43) follows. Next, using (15) and after some algebra, the inverse of $F_{\Gamma_{\min }}^{\mathrm{II}}(\gamma)=q$ becomes

$$
F_{\Gamma_{\min }}^{\mathrm{II}^{-1}}(q)=\left(\bar{\kappa}+\delta_{\kappa}\left(\sqrt{1-q}-\frac{1}{2}\right)\right)^{-2} .
$$

Finally, using Lemma 4, the normalization coefficients are obtained as in (44). 


\section{REFERENCES}

[1] M. Kazemi and A. Mohammadi, "Scalability analysis of DF relay selection in massive MIMO systems with hardware impairments," in Proc. 9th Int. Symp. Telecom., 2018, pp. 149-154.

[2] T. L. Marzetta, "Noncooperative cellular wireless with unlimited numbers of base station antennas," IEEE Trans. Wireless Commun., vol. 9, no. 11, pp. 3590-3600, Nov. 2010.

[3] E. Larsson, O. Edfors, F. Tufvesson, and T. Marzetta, "Massive MIMO for next generation wireless systems," IEEE Commun. Mag., vol. 52, no. 2, pp. 186-195, Feb. 2014

[4] L. Lu, G. Y. Li, A. L. Swindlehurst, A. Ashikhmin, and R. Zhang, "An overview of massive MIMO: Benefits and challenges," IEEE J. Sel. Topics Signal Process., vol. 8, no. 5, pp. 742-758, Oct. 2014.

[5] J. Choi, J. Mo, and R. W. Heath, "Near maximum-likelihood detector and channel estimator for uplink multiuser massive MIMO systems with one-bit ADCs," IEEE Trans. Commun., vol. 64, no. 5, pp. 2005-2018, May 2016

[6] M. Kazemi, H. Aghaeinia, and T. M. Duman, "Discrete-phase constant envelope precoding for massive MIMO systems," IEEE Trans. Commun., vol. 65, no. 5, pp. 2011-2021, May 2017.

[7] X. Zhang, M. Matthaiou, M. Coldrey, and E. Björnson, "Energy efficiency optimization in hardware-constrained large-scale MIMO systems," in Proc. 11th Intl. Symp. Wireless Commun. Syst., 2014, pp. 992-996.

[8] X. Zhang, M. Matthaiou, M. Coldrey, and E. Björnson, "Impact of residual transmit RF impairments on training-based MIMO systems," IEEE Trans. Commun., vol. 63, no. 8, pp. 2899-2911, Aug. 2015.

[9] N. H. Nhat, V. N. Q. Bao, N. Linh Trung, and M. Debbah, "Relay selection in two-way relaying networks with the presence of HWI at relay transceiver," in Proc. Intl. Conf. Adv. Tech. Commun., Hanoi, 2014, pp. 616-620.

[10] K. Guo, J. Chen, and Y. Huang, "Outage analysis of cooperative communication network with HWI," Frequenz, vol. 69, no. 9-10, pp. 443-449, 2015.

[11] K. Guo, B. Zhang, Y. Huang, and D. Guo, "Outage analysis of multi-relay networks with hardware impairments using SECps scheduling scheme in shadowed-Rician channel," IEEE Access, vol. 5, pp. 5113-5120, 2017.

[12] K. Guo, D. Guo, and B. Zhang, "Performance analysis of two-way multiantenna multi-relay networks with hardware impairments," IEEE Access, vol. 5, pp. 15971-15980, 2017.

[13] N. T. Do, D. B. da Costa, and B. An, "Performance analysis of multirelay RF energy harvesting cooperative networks with hardware impairments," IET Commun., vol. 10, no. 18, pp. 2551-2558, Dec. 2016.

[14] P. K. Sharma and P. K. Upadhyay, "Cognitive relaying with transceiver hardware impairments under interference constraints," IEEE Commun. Lett., vol. 20, no. 4, pp. 820-823, Apr. 2016.

[15] E. Balti, M. Guizani, B. Hamdaoui, and B. Khalfi, "Aggregate hardware impairments over mixed RF/FSO relaying systems with outdated CSI," IEEE Trans. Commun., vol. 66, no. 3, pp. 1110-1123, Mar. 2018.

[16] V. P. Tuan and H. Y. Kong, "Impact of residual transmit RF impairments on energy harvesting relay selection systems," Intl. J. Electron., vol. 104 no. 6, pp. 928-941, 2017.

[17] E. Björnson, M. Matthaiou, and M. Debbah, "A new look at dual-hop relaying: Performance limits with hardware impairments," IEEE Trans. Commun., vol. 61, no. 11, pp. 4512-4525, Nov. 2013.

[18] M. Matthaiou, A. Papadogiannis, E. Björnson, and M. Debbah, "Two-way relaying under the presence of relay transceiver hardware impairments,' IEEE Commun. Lett., vol. 17, no. 6, pp. 1136-1139, Jun. 2013.

[19] H. A. David and H. N. Nagaraja, Order Statistics, 3rd ed., Hoboken, NJ, USA: Wiley, 2003.

[20] E. Castillo, Extreme Value Theory in Engineering. New York, NY, USA: Academic, 1988.

[21] A. BenMimoune and M. Kadoch, "Relay technology for 5G networks and IoT applications," in Internet of Things: Novel Advances and Envisioned Applications. Berlin, Germany: Springer, 2017, pp. 3-26.

[22] D. Zhang, Y. Liu, Z. Ding, Z. Zhou, A. Nallanathan, and T. Sato, "Performance analysis of non-regenerative massive-MIMO-NOMA relay systems for 5G," IEEE Trans. Commun., vol. 65, no. 11, pp. 4777-4790, Nov. 2017.

[23] S. Jin, X. Liang, K. Wong, X. Gao, and Q. Zhu, "Ergodic rate analysis for multipair massive MIMO two-way relay networks," IEEE Trans. Wireless Commun., vol. 14, no. 3, pp. 1480-1491, Mar. 2015.

[24] A. Papoulis and U. Pillai, Probability, Random Variables, and Stochastic Processes, 4th ed. New York, NY, USA: McGraw-Hill, 2002.

[25] A. M. Tulino and S. Verdú, Random Matrix Theory and Wireless Communications. Boston, MA, USA: Now, 2004.
[26] K. R. Liu, A. K. Sadek, W. Su, and A. Kwasinski, Cooperative Communications and Networking. Cambridge, U.K.: Cambridge Univ. Press, 2009

[27] A. Kuhestani, A. Mohammadi, K. Wong, P. L. Yeoh, M. Moradikia, and M. R. A. Khandaker, "Optimal power allocation by imperfect hardware analysis in untrusted relaying networks," IEEE Trans. Wireless Commun., vol. 17 , no. 7, pp. 4302-4314, Jul. 2018.

[28] E. Björnson, M. Matthaiou, and M. Debbah, "A new look at dual-hop relaying: performance limits with hardware impairments," IEEE Trans. Commun., vol. 61, no. 11, pp. 4512-4525, Nov. 2013.

[29] E. Björnson, J. Hoydis, M. Kountouris, and M. Debbah, "Massive MIMO systems with non-ideal hardware: Energy efficiency, estimation, and capacity limits," IEEE Trans. Inf. Theory, vol. 60, no. 11, pp. 7112-7139, Nov. 2014

[30] H. Holma and A. Toskala, LTE for UMTS: Evolution to LTE-Advanced, 2nd ed., Hoboken, NJ, USA: Wiley, 2011.

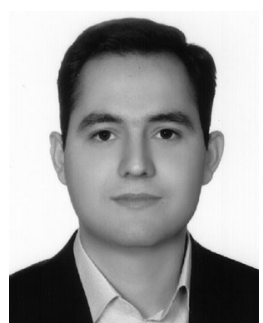

Mohammad Kazemi (Member, IEEE) received the B.S. and M.S. degrees from the K.N. Toosi University of Technology, Tehran, Iran, in 2007 and 2010, respectively, and the $\mathrm{Ph} . \mathrm{D}$. degree from the Amirkabir University of Technology, Tehran, Iran, in 2017, all in electrical engineering. He was a Visiting Student with Bilkent University, Turkey, in 2015. He was a Researcher in MMWCL with the Amirkabir University of Technology from 2017 to 2019 . He is currently a Postdoctoral Fellow with the EE Department, Bilkent University, Ankara, Turkey. His research interests are in wireless communications and signal processing, with particular focus on massive MIMO and massive random access systems. He is currently an Editorial Assistant to the Editor-in-Chief forIEEE TRANSACTIONS ON COMMUNICATIONS.

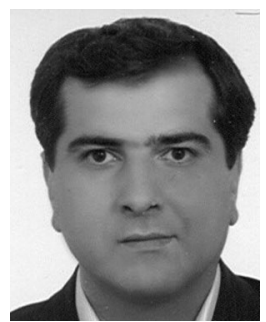

Abbas Mohammadi (Senior Member, IEEE) received the B.Sc. degree in electrical engineering from Tehran University, Tehran, Iran, in 1988, and the M.Sc. and Ph.D. degrees in electrical engineering from the University of Saskatchewan, Saskatoon, SK, Canada, in 1995 and 1999, respectively. In 1998, he joined Vecima Networks Inc., Victoria, Canada, as a Senior Research Engineer where he conducted research on Wireless Communications. Since March 2000, he has been with the Electrical Engineering Department, Amirkabir University of Technology (Tehran Polytechnic), Tehran, Iran, where he is currently a Professor. He has been an ICORE Visiting Professor in Electrical and Computer Engineering Department of the University of Calgary, Canada and Nokia Visiting Professor with Tampere University of Technology, Finland. He has authored more than 200 Journal and Conference papers and holds three U.S. and one Canadian Patents. He has coauthored, Six-port Technique with Microwave and Wireless Applications, (Artech House, 2009) and RF Transceiver design for MIMO Wireless Communications (Springer, 2012). His current research interests include broadband wireless communications, adaptive modulation, MIMO systems, mesh and ad hoc networks, microwave and wireless subsystems, software design radio, and advanced wireless transceiver architectures.

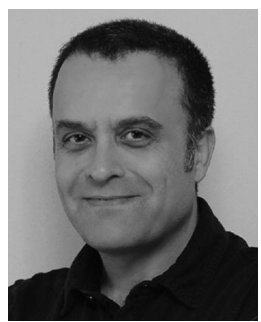

Tolga M. Duman (Fellow, IEEE) received the B.S. degree from Bilkent University, Ankara, Turkey, in 1993, and the M.S. and Ph.D. degrees from Northeastern University, Boston, MA, USA, in 1995 and 1998 , respectively, all in electrical engineering. He is a Professor of Electrical and Electronics Engineering Department, Bilkent University, in Turkey. Prior to joining Bilkent University in September 2012, he was with the School of ECEE, Arizona State University as an Assistant Professor from 1998 to 2004, an Associate Professor from 2004 to 2008, and a Professor after 2008. His current research interests are in systems, with particular focus on communication and signal processing, including wireless and mobile communications, coding/modulation, coding for wireless communications, data storage systems, and underwater acoustic communica- tions. He is the recipient of the National Science Foundation CAREER Award and the IEEE Third Millennium medal, and he is currently an Editor-in-Chief for IEEE TRANSACTIONS ON COMMUNICATIONS 